\title{
Large Antenna Array with Hybrid Beamforming System for 5G Indoor Communication Network Deployments
}

Jeyakumar $P$ ( $\nabla$ jeyakumarpsg@gmail.com )

National Institute of Technology Tiruchirappalli https://orcid.org/0000-0002-2043-790X

Malar E

PSG Institute of Technology and Applied Research

Srinitha S

Sri Ramakrishna Engineering College

Muthuchidambaranathan $\mathrm{P}$

National Institute of Technology Tiruchirappalli

Arvind Ramesh Ramesh

NIT-Tiruchirappalli: National Institute of Technology Tiruchirappalli

\section{Research Article}

Keywords: mmWave, antenna array, hybrid beamforming, link budget, $5 \mathrm{G}$ point-to-point communications

Posted Date: May 21st, 2021

DOl: https://doi.org/10.21203/rs.3.rs-497933/v1

License: (9) This work is licensed under a Creative Commons Attribution 4.0 International License. Read Full License 


\title{
Large Antenna Array with Hybrid Beamforming System for 5G Indoor Communication Network Deployments
}

\author{
Jeyakumar $\mathbf{P}^{1}$, Malar $\mathbf{E}^{2}$, Srinitha $\mathbf{S}^{2}$, \\ Muthuchidambaranathan $\mathbf{P}^{1}$, Arvind \\ Ramesh $^{1}$
}

Received: date / Accepted: date

\begin{abstract}
The millimeter-wave multiple input multiple output (MIMO) technology is the frontier for $5 \mathrm{G}$ communication systems. This work contributes a large antenna array with a limited number of radio frequency chains using the hybrid beamforming ( $\mathrm{HBF}$ ) technique that overcomes extreme path loss in the mmWave system to improve spectral efficiency. The link budget analysis is given for the target data rate of $11.3 \mathrm{Gbps}$ for the point-to-point communication. The number of antenna elements required for the proposed antenna array is determined via link budget analysis. The proposed system includes single element patch antenna configuration, array factor analysis, and beam steering capability. The transmit and receive antenna gain specifications minimize the path loss and improve the system throughput. Combiners and hybrid precoders are designed together in an iterative way for reducing the
\end{abstract}

Jeyakumar $\mathrm{P}^{1}$

Department of Electronics and Communication Engineering,

National Institute of Technology, Tiruchirappalli 620015, India

E-mail: jeyakumarpsg@gmail.com

Malar $\mathrm{E}^{2}$

Department of Electrical and Electronics Engineering,

PSG Institute of Technology and Applied Research, India

E-mail: emr@psgitech.ac.in

Srinitha $\mathrm{S}^{2}$

Department of Electronics and Communication Engineering,

Sri Ramakrishna College of Engineering, Coimbatore, India

E-mail: srinitha@srec.ac.in

Muthuchidambaranathan $\mathrm{P}^{1}$

Department of Electronics and Communication Engineering,

National Institute of Technology, Tiruchirappalli 620015, India

E-mail: muthuc@nitt.edu

Arvind Ramesh ${ }^{1}$

Department of Instrumentation and Control Engineering,

National Institute of Technology, Tiruchirappalli 620015, India

E-mail: arvindramesh25@gmail.com 
cost function of the weighted minimum mean squared(WMMSE) error. Simulation results demonstrate that the proposed HBF algorithm performance is highly effective and performs closer to the fully digital beamforming technique. The proposed large antenna array with HBF uses the New York University Simulator (NYUSIM) to perform omnidirectional and directional power delay profile analysis with the most potent power. The proposed large antenna array with HBF methodology provides an optimal approach to indoor point-to-point communication deployments.

Keywords mmWave, antenna array, hybrid beamforming, link budget, 5G point-to-point communications

\section{Introduction}

The frequency spectrum of $300 \mathrm{MHz}-3 \mathrm{GHz}$ is simultaneously used by most wireless communication networks, even though the bandwidth of the $3 \mathrm{GHz}$ $300 \mathrm{GHz}$ frequency range is not entirely used [1]. This is generally because the low-frequency transmission allows penetration of radio waves across buildings, multiple wave reflection, and adjustable bending around the corners. The 30 $\mathrm{GHz}-300 \mathrm{GHz}$ spectrum, known as the mmWave band, has a $(1-10) \mathrm{mm}$ wavelength range. This mmWave frequency band is proper in many real-world applications because it facilitates high data rates for larger spectral channels [2]. The mmWave is associated with high ambient absorption, heavy losses due to penetration and reflection, attenuation owing to rain and foliage, and slight diffraction. All the aspects mentioned above restrict them to relatively small Line of Sight (LOS) in indoor-to-indoor and outdoor-to-outdoor communication. The major bottleneck of employing mmWaves is its limited range of frequency. A cost-effective alternative for wired communication systems and even wireless local area networks (WLAN) depending upon the IEEE 802.11ad protocol is given by $60 \mathrm{GHz}$ mmWave band of unlicensed wireless backhaul band [3]. Having the current theoretical considerations and measurement campaigns into account, it is evident that, if both the transmitter and receiver are outfitted adequately with "large" antenna arrays, then the small outdoor cells with $200 \mathrm{~m}$ of cell radii is feasible to neutralize the effect of propagation losses [4].

From the literature, it is evident that many radiating components are used extensively in an antenna to provide efficient mmWave communication. This is described by the transmission formula given by Friis [5], which explains the relation between the transmitter power $\left(P_{t}\right)$ and the receiver power $\left(P_{r}\right)$ for the two antennas separated by a distance 'd' under optimum conditions and the free space propagation is given in equation (1).

$$
\frac{P_{r}}{P_{t}}=G_{r} G_{t}\left(\frac{\lambda}{4 \pi d}\right)^{2}
$$

$\lambda$ is the wavelength, and $G_{t}$ and $G_{r}$ denotes the gains of both transmitter and receiver antennas. In equation (1), an assumption is made that these antennas 
are aligned perfectly. If $G_{t}, G_{r}$ is fixed, then the path loss $P_{t} / P_{r}$ is proportional to $\lambda^{-2}$. This implies that if there are no directional antenna gains, then the mmWave suffers from a larger path loss. Consequently, at mmWave frequencies, the increase in free-space path loss is compensated more by scaling antenna gains. This compensation process demands a directional transmission is having large antenna arrays of high dimension [5]. However, the deployment of large antenna elements presents some challenges in power consumption, size, and cost. Also it is not possible to equip the each antennas with the distinct Radio Frequency (RF) chain with the help of today's technology [6]. Specifically, owing to the parallel data processing of large volumes of data with the sampling speed of millions of samples per second, analog to digital converters (ADCs), the power amplifiers, digital to analog converters (DACs) consumes more power at mmWave frequencies [7]. Alternatively, suppose digital beamforming techniques are taken into account. In that case, a separate RF chain is required for any antenna element in a large antenna array, which paves the way for enormous costs and high power consumption [8]. Therefore, in this study, to reduce high power consumption and overcome path loss, hybrid beamforming $(\mathrm{HBF})$ is proposed with a uniform linear array (ULA) considered an alternate approach, as shown in Figure 1.

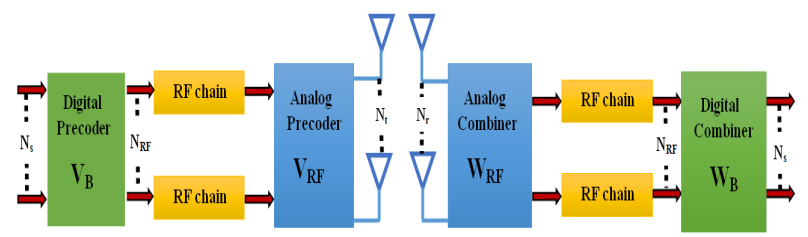

Fig. 1 Point-to-Point mmWave Communication

\subsection{Related Work}

Nowadays, mmWave communications have drawn considerable research interest, as the immense bandwidth available would theoretically lead to several Gbps (gigabit per second) rates per user [8]. Beamforming in outdoor mmWave systems would be vital to address high path losses and achieve fair link budgets [10]. The analog RF beam formation solutions suggested in previous works were highly dependent on the phase regulation of the transmitted signal through the analog phase shifter network by each antenna [13] - [15]. But, owing to the shortcomings of the analog beamforming hardware, the efficiency of the analog techniques proposed is usually sub-optimal. Fully digital and hybrid analog/digital processing methodologies were introduced for achieving more significant beamforming benefits and for allowing precoding of multiple data streams [16],[17]. 
As HBF is compared with the conventional fully digital beamforming approach, HBF poses complexity by transmitting, receiving, analog, and digital beamforming variables to achieve an optimum design [10]. And the constant modulus conditions of analog beam-formers present in phase shifters produce a complex, non-convex problem that is very difficult to resolve [18]. This complexity can be minimized by the following method. Initially, the exact problem is decoupled into hybrid precoding, and all sub-problems are combined, and then module conditions are applied to solve the sub-problems. The HBF process is considered to be a matrix factorization, and the Euclidean distance between the fully digital beam-former and the hybrid beam-former is reduced $[20],[22],[24]$. The spatial structure of mmWave propagation channels is suggested to overcome this matrix factorization [20]. The proposed precoding technique and combinatorial algorithms use Orthogonal Matching Pursuit (OMP) [21]. In addition to using the method of matrix factorization, the exact problem can also be explicitly discussed. Manifold Optimization and non-complex algorithms are also suggested with the HBF algorithm [22].

For mmWave MIMO systems, the hybrid RF/baseband linear precoding design using an optimal linear equalizer was proposed, aiming to minimize the Euclidean distance between the hybrid precoder and the optimal minimum MSE precoder. Low-complexity and numerically robust Jacobi algorithms were used to achieve relatively close results to that of the optimal design [24]. Similarly, for each range of error weights, an optimal linear precoder and decoder are built to diagonalize the MIMO channel into Eigen subchannels using the weighted MMSE criteria subject to the transmitter power limit [25]. Linear precoders and equalizers were made to derive an efficient beam vector with minimal BER for multi-carrier MIMO systems [26]. The system was constructed using Schur-concave and Schur-convex functions, such that the channel diagonalizing structure is often optimal for Schur-concave objective functions. In contrast, the optimal solution for Schur-convex functions only diagonalizes the channel after the particular transmitted symbol rotation.

All the preceding works related to the design of HBF aims to maximize the spectrum efficiency. In addition to spectral efficiency, Mean Square Error (MSE) is also an essential metric in fully-digital MIMO systems [25]. A system is guarded with specific modulation techniques and coding methods rather than Gaussian code [26], but MSE acts as a direct metric to measure the reliability during transmission. Modified-MSE, Sum-MSE, Min-max-MSE are some of the variants of MSE that are pertinent to other performance metrics like SINR (Signal to Interference plus Noise Ratio) and SER (Symbol Error Rate) [25], [28], [29]. From existing works, it is found that the MSE is pertinent to Bit Error Rate (BER) and SINR in designing fully digital beam-formers in MIMO systems[25], [26]. Therefore MSE acts as an essential optimization metric in designing HBF. In the prevailing HBF design methodologies, having the goal of increasing the spectral efficiency, by minimizing the MSE, the hybrid receives combining matrices were optimized [20], [23], and [30]. From the preceding works, it is also evident that the precoding design using the MMSE (Minimum MSE) condition shows significant results in terms of spectral effi- 
ciency [30], [32], and [33]. By comparing other conventional techniques [12], [29], and [30], the proposed methodology using the WMMSE criterion for HBF designing to improve spectral efficiency.

\subsection{Major Contributions}

The followings are the proposal's major contribution :

- The first link budget analysis is calculated for short-range communication, including transmitting power, transmitting antenna gain, receiving antenna gain and receiving power, etc. The framework is then configured to consider factors such as the number of antenna elements for source and destination specifications.

- Combiners and precoders are developed iteratively for a reduced cost function. Consequently, the spectral performance and system throughput are greatly improved by implementing the proposed HBF algorithm using the WMMSE criterion. By this method, the spectral efficiency is improved and the bit error rate (BER) is reduced.

The rest of this article is structured as follows: Section II includes an overview of the link budget to illustrate the communication system's feasibility. Section III tells about the microstrip patch antenna design and large-scale antenna array design. Depth the proposed design technique for HBF is discussed in Section IV. The simulation results, system complexity analysis, and system throughput assessment in Section V. Finally, Section VI concludes the article.

\section{Link Budget Analysis}

The required power at the transmitter and the receiver should be adjusted carefully for establishing the communication link between the transmitter and the receiver and also maintain the desired data rate of a channel. The power analysis is essential to create a link that relies upon the link budget. This link budget depends on the specifications of both source and destination, namely SNR (Signal to Noise Ratio), transmitting power, Gain of the antenna, and throughput of the established link. Table 1 denotes the proposed link budget for the point-to-point $60 \mathrm{GHz}$ mmWave communication systems. The carrier frequency of $60 \mathrm{GHz}$ is chosen with $30 \mathrm{dBm}$ transmit power at the base station. The path loss model described in equation (2) suits best for LOS short-range wireless communication systems described by Hemadeh et al. [34].

Path Loss and Shadowing Model:

The mean path loss model described by Hemadeh et al is given by $(2)$

$$
P L(d)[d B]=P L_{0}\left(d_{0}\right)+10 n \log _{10}\left(\frac{d}{d_{0}}\right)-S_{\sigma},
$$


Table 1 The link budget for $60 \mathrm{GHz}$ Communication

\begin{tabular}{|c|c|c|}
\hline Transmit power $(\mathrm{dBm})$ & 30 & $\mathrm{a}$ \\
\hline Transmit antenna gain $(\mathrm{dBi})$ & 25 & $\mathrm{~b}$ \\
\hline Carrier frequency $(\mathrm{GHz})$ & 60 & - \\
\hline Distance $(\mathrm{m})$ & 200 & - \\
\hline Losses $(\mathrm{dB})$ & 20 & $\mathrm{c}$ \\
\hline Path loss $(\mathrm{dB})$ & 117.05 & $\mathrm{~d}$ \\
\hline Receive antenna gain $(\mathrm{dBi})$ & 25 & $\mathrm{e}$ \\
\hline Bandwidth $(\mathrm{GHz})$ & 2 & - \\
\hline Receiver Power $(\mathrm{dBm})$ & -57.05 & $\mathrm{f}=\mathrm{a}+\mathrm{b}+\mathrm{e}-\mathrm{c}-\mathrm{d}$ \\
\hline Noise PSD $(\mathrm{dBm} / \mathrm{Hz})$ & -174 & - \\
\hline Noise figure(db) & 10 & - \\
\hline Thermal Noise $(\mathrm{dBm})$ & -71 & $\mathrm{~g}$ \\
\hline SNR $(\mathrm{dB})$ & 13.95 & $\mathrm{~h}=\mathrm{f}-\mathrm{g}$ \\
\hline Implementation Loss $(\mathrm{dB})$ & 3.00 & - \\
\hline Spectral efficiency & 5.65 & $\log _{2}(1+S N R)$ \\
\hline Target Data rate $(\mathrm{Gbps})$ & 11.3 & $\mathrm{BW}{ }^{*} \mathrm{SE}$ \\
\hline
\end{tabular}

where d denotes the separation between the transmitter and the receiver, $\mathrm{n}$ is the path loss exponent parameter, $P L_{0}$ indicates the path loss of free space concerning the reference distance $d_{0}$ which is one meter for the indoor environments. $\sigma$ is the standard deviation parameter of variations and $S_{\sigma}$ of the signal power average measured in decibels. The statistical parameters of 60 GHz LOS channel path-loss model are $P L_{0}=68 d B, n=2.17$ and $S_{\sigma}=0.88$. Using equation (2), in a LOS environment with a standard deviation of 0.88 $\mathrm{dB}$, the path loss is calculated to be $117.05 \mathrm{~dB}$ over a distance of $200 \mathrm{~m}$. The primary goal of this system design is to attain $11.3 \mathrm{Gbps}$ of data rate with a bandwidth of $2 \mathrm{GHz}$ to 200 meters Inter-Site Distance (ISD) operating in the $60 \mathrm{GHz}$ band. The link of $11.3 \mathrm{Gbps}$ throughput is effectively formed by using Shannon's channel capacity, and $13.95 \mathrm{~dB}$ of SNR is required [35]. In addition to considering the device losses due to impedance mismatch, an extra $3 \mathrm{~dB}$ loss is added to consider DAC / ADC and circuit power dissipation. Therefore, to build the link, a total of $16.95 \mathrm{~dB}$ of SNR is required. Thermal noise of -174 $\mathrm{dBm} / \mathrm{Hz}$ and a noise figure of $10 \mathrm{~dB}$ is technically assumed. It is also believed that $8 \mathrm{dBi}$ of each separate element using microstrip patch antennas can also achieve the same $25 \mathrm{dBi}$ of transmitting and receive antenna gain. Hence, the number of antennas needed to compensate for the path loss with the help of the link budget is clarified in the next section.

\section{Antenna Element Analysis}

\subsection{Patch Antenna Design}

The design of a single microstrip patch antenna operating at $60 \mathrm{GHz}$ point-topoint communications is depicted in Fig 2. Quarter-Wave Transformer (QWT) methodology is used to improve impedance matching. Antenna parameters 
Table 2 Antenna Parameters Summary

\begin{tabular}{|l|l|}
\hline Patch Parameters & Value $(\mathrm{mm})$ \\
\hline Patch Length(L) & 1.521 \\
\hline Patch Width(W) & 1.84 \\
\hline QWT width & 0.11 \\
\hline QWT length & 1.605 \\
\hline
\end{tabular}

such as length, the width of the antenna, QWT length, and width of feed-lines are shown in Table 2. By using the HFSS software, the return loss plot of a single element of the micro-strip patch antenna is obtained as shown in fig 3, and it is estimated as $-15.32 \mathrm{~dB}$. The band-width of the micro-strip antenna is $1500 \mathrm{MHz}$ which meet the requirements that is listed in the Table 1.

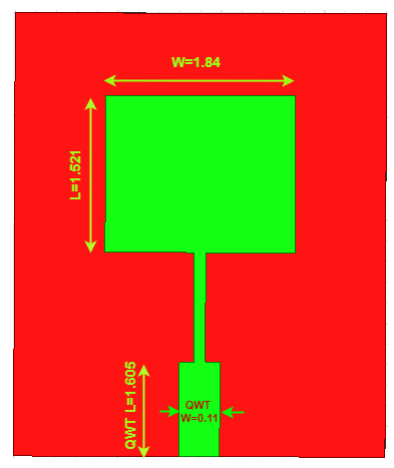

Fig. 2 Single Microstrip Patch antenna

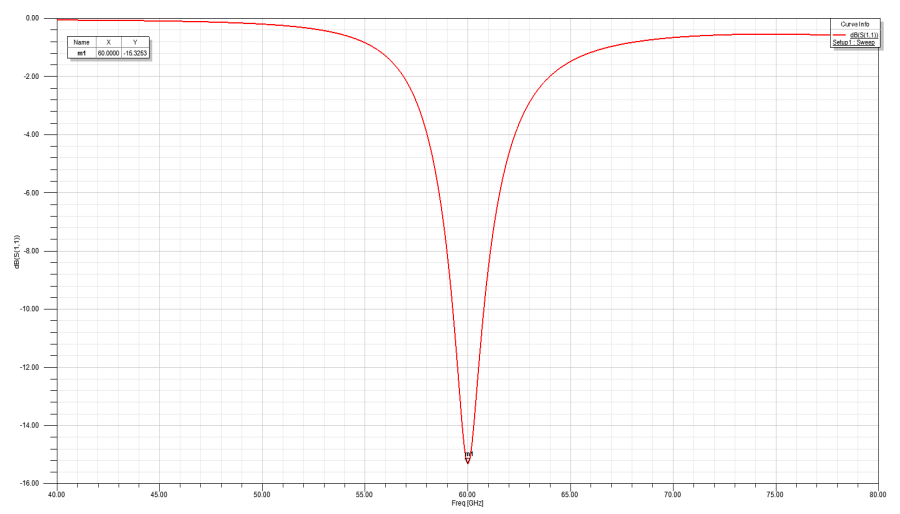

Fig. 3 Return loss graph of the single patch antenna at $60 \mathrm{GHz}$ carrier frequency 
Table 3 Simulation results

\begin{tabular}{|c|c|}
\hline Simulation Parameters & Single Patch Antenna \\
\hline Return loss & -15.32 \\
\hline Bandwidth & $1500 \mathrm{MHz}$ \\
\hline Directivity & $8.15 \mathrm{dBi}$ \\
\hline Gain & $8.19 \mathrm{dBi}$ \\
\hline HPBW(E-Plane) & $83.4^{\circ}$ \\
\hline HPBW(H-Plane) & $89.8^{\circ}$ \\
\hline
\end{tabular}

The single element antenna's radiation pattern is depicted in Fig 4. It is evident from the radiation pattern that $-3 \mathrm{~dB}$ values for E-plane and $\mathrm{H}$-plane beamwidth are $83.4^{\circ}$ and $89.8^{\circ}$ respectively. The single element antenna's gain plot is represented in Fig.5 The simulation results of single element patch design is tabulated in Table 3 . This table represents the half power beamwidth (HPBW) for both H-Plane and E-plane, gain and directivity extracted from the radiation pattern.

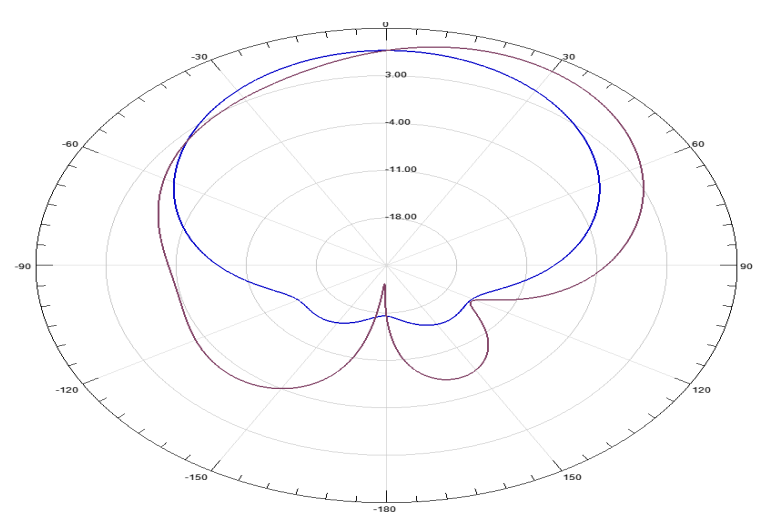

Fig. 4 Radiation pattern of the single patch antenna for H-plane and E-plane

\subsection{Antenna Array Factor Analysis}

At the base station, the gain of an antenna is assumed to be $8 \mathrm{dBi}$, as an antenna with low gain can provide high steering capability and minimum power leakage at the side lobes [34]. Consequently, $17 \mathrm{dBi}$ of gain is needed in surplus to attain $11.3 \mathrm{Gbps}$ of data rate. Here, the total gain of an antenna array is given by $G_{\text {antenna }}(3)$ with the spacing of $d=\lambda / 2$

$$
G_{\text {antenna }}=\eta G_{\text {element }} N
$$




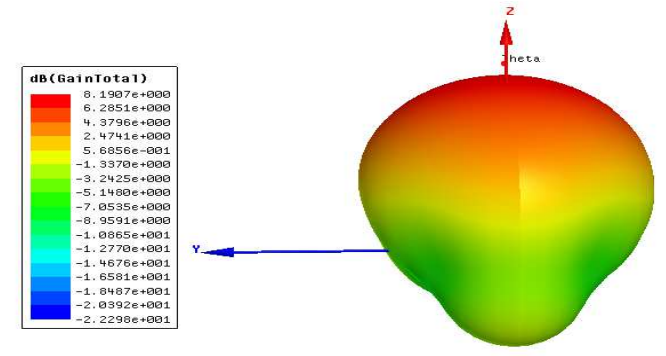

Fig. 5 Gain response of the single element antenna

Where $\eta$ represents an antenna array competence to steer the beam exclusively on the desirable direction. The gain of an antenna $G_{\text {antenna }}$ is also represented as the summation of both array gain and an element gain.

$$
G_{\text {antenna }}(d B i)=10 \log \eta+G_{\text {element }}(d B i)+\operatorname{Arraygain}(d B) .
$$

Subsequently, a gain of $17 \mathrm{dBi}$ is obtained in the surplus from an array. In order to acquire $17 \mathrm{dBi}$ gain from an array having $\eta=1,\left(10^{\frac{17}{10}}\right) \approx 50$ antenna elements are needed. Meanwhile, in general, $\eta \neq 1$, as there are significant radiations from other directions, especially during steering. Practically, it is not feasible to have a narrow pencil beam to attain high gain from antenna arrays. To attain high integrity, it is essential to have more than 32 antenna elements based on the antenna array's directivity. For $\eta=0.75$, nearly 67 antenna elements are needed to attain $17 \mathrm{dBi}$ of gain. Correspondingly, for $\eta=0.5$, around 100 antenna elements are needed. After designing the single antenna element, it is converted into 'N' ULA (Uniform Linear Array) with equal spacing. The radiation pattern of the single element linear antenna array is relatively approximated to the value which is obtained by multiplying the array factor $A F_{\text {array }}$ with the 100 antenna element radiation pattern denoted by $F_{\text {array }}$ that is pretended to be equivalent for all antenna array elements.

The ULA array factor is expressed in the equation (5)

$$
F_{\text {array }}(\theta, \phi)=F_{\text {element }}(\theta, \phi) \times A F_{\text {array }}(\theta, \phi)
$$

For a small number of antenna elements, an assumption of having equal radiation pattern does not hold good. There will be a large degree of deviation of outer antenna elements from the radiation patterns of other antenna that cannot be discarded in the event of having only small number of antenna elements. However, this will be reasonable for gross approximations. In the equation (5), the losses present in the antenna elements and mutual coupling is not taken into account. This kind of effects will result in a formation of reformed beam patterns that can be manifested to increase the levels of side 


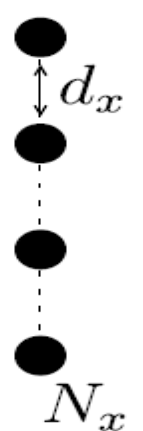

Fig. 6 Linear array arrangement

lobes. To estimate the value of $F_{\text {array }}$, the value of $A F_{\text {array }}$ is indispensable. The array factor in (6) depends on the number of antenna elements ' $\mathrm{N}$ ', the angle direction $\theta$, the wavelength $\lambda$ and the distance between the antenna elements 'd' [36].

$$
A F_{\text {array }}(\theta, \phi)=\Sigma_{n=1}^{N} a_{n} e^{j n k d \sin \theta \sin \phi} e^{\Delta \varphi} ; k=2 \times \pi / \lambda
$$

By using the above expression (6), the complex weights are determined in the beam-forming process and the angle $\Delta \varphi$ can be estimated. The factor $a_{n}$ denotes the amplitude weights that are applied to each antenna element. The angle $\Delta \varphi$ represents the beam steering which can be shifted to the desirable angle.

$$
\Delta \phi=\frac{2 \pi d \sin \theta}{\lambda}
$$

The equation (6) is generalised by imparting $\varphi$ that represents the far zone phase-difference of the nearby adjacent elements.

$$
\psi=k d \sin \theta \sin \phi+\Delta \varphi
$$

Substituting (8) in (6)

$$
A F_{\text {array }}(\theta, \phi)=\Sigma_{n=1}^{N} a_{n} e^{j n \psi}
$$

The equation (9) is simplified further and normalized. The array factor after simplification is given by (10).

$$
\left|A F_{\text {array }}(\psi)\right|=\frac{1}{N}\left|\frac{\sin (n \psi / 2)}{\sin (\psi / 2)}\right|
$$

The normalized $A F_{\text {array }}$ is periodic in $[0,2 \pi]$ interval and it permits to obtain the details of linear antenna array characteristics.

Fig. 7 represents the proposed 100 element antenna radiation pattern of ULA with $28.16 \mathrm{dBi}$ of array gain and spacing of $0.5 \lambda$ among each antenna 


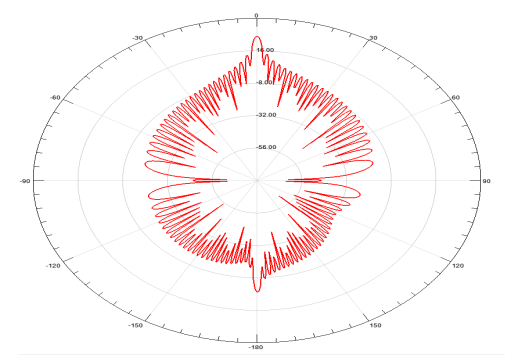

Fig. 7 Radiation pattern of the proposed Uniform Linear Array[ $\mathrm{N}=100]$

element. From Table 1, it is evident that the horizontal beam-width of an antenna $\mathrm{HPBW}$ is $\theta a=10^{\circ}$ which is highly adequate to cover an area (> $200 m)$.

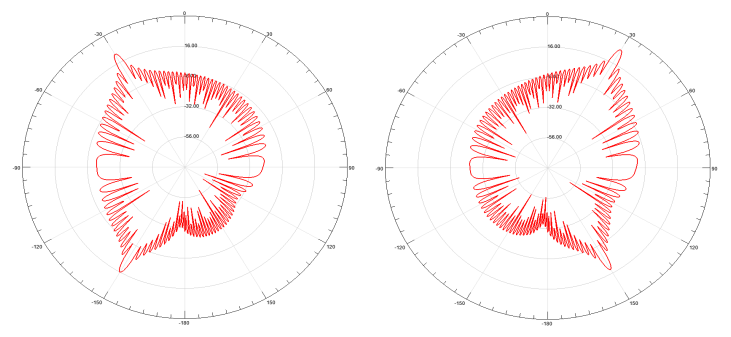

Fig. 8 Beam Steering at $\pm 15^{\circ}$

At $60 \mathrm{GHz}$ frequency, the main beam of an antenna is steered at an angle of $\pm 15^{\circ}$. By using equation (7), $44.99^{\circ}$ of phase offset is attained. Here, the array gain is $27.78 \mathrm{dBi}$. As a consequence of steering, the gain-loss is mitigated and is less than $0.38 \mathrm{dBi}$. Hence, the suggested 100-element array antenna attained a very narrow pencil beam having $\pm 15^{\circ}$ as its maximum beam steering that is used as specified by standards requirements [37].

\section{Hybrid Beamforming}

consider a point-to-point mmWave communication system having HBF precoder blocks at the source and the destination, as represented in Fig. 1. The number of receiving and transmitting antennas is $N_{r}$ and $N_{t}$, respectively. $N_{s}$ data streams are sent digital linear precoder. The number of transmitting and receiving antennas should be larger than the number of outfitted RF chains denoted by $N_{R F}$. i.e., $N_{R F} \ll \min \left(N_{r}, N_{t}\right)$. The symbol vector has the size of $N_{s} \times 1$ together with $E\left[s s^{H}\right]=I$ will be precoded with both analog and digital beamforming matrices. The digital beamforming matrix is indicated by $V_{B}$ and has the size of $N_{R F} \times N_{s}$. Analog beamforming matrix is indicated 
by $V_{R F}$ and has the size of $N_{t} \times N_{R F}$. The precoded signal vector is denoted by $x=V_{R F} V_{B} s$ in contrast to the equivalent base-band representation at the transmit antenna array. The normalized transmit power constraint is fixed to $\operatorname{tr}\left(V_{R F} V_{B} V_{B}^{H} V_{R F}^{H}\right) \leq 1$ without any loss in its generality.

Notations: Lower-case and upper-case boldface letters denote vectors and matrices, respectively. $(.)^{T},(.)^{H},(.)^{-1}$, and $\operatorname{det}($.$) denote the transpose, con-$ jugate transpose, inversion, and determinant of a matrix, respectively; $\|\cdot\|_{2}$ denotes the 2-norm of a vector. $|\cdot|$ denote the absolute operator; Re. and Im. denote the real part and imaginary part of a complex number, respectively; $\mathrm{E}($.$) represents the expectation; Finally, I_{N}$ is the $N \times N$ identity matrix.

\section{$4.1 \mathrm{mmWave}$ Channel model}

The mmWave propagation channel consists of $N_{c}$ number of clusters in which each of the single cluster consists of $N_{R}$ number of rays which is similar to the existing works [12] \& [20]. In this system model, half wave spaced ULA is considered at the transmitter and receiver side with the channel matrix H. Its size $N_{r} \times N_{t}$ is denoted by the following equation (11)

$$
H=\sqrt{\frac{N_{t} N_{r}}{N_{C} N_{R}}} \sum_{i=1}^{N_{C}} \sum_{j=1}^{N_{R}} \alpha_{i j} a_{r}\left(\theta_{i j}^{r}\right) a_{t}\left(\theta_{i j}^{t}\right)^{H}
$$

where $\theta_{i j}^{r}$ represents the arrival angle, $\theta_{i j}^{t}$ represents the departure angle, $\alpha_{i j}$ denotes the complex gain of the jth ray which is present in the ith propagation cluster and $a_{r}\left(\theta_{i j}^{r}\right)=\frac{1}{\sqrt{N_{r}}}\left[1, e^{j \pi \sin \theta_{i j}^{r}} \ldots e^{j \pi\left(N_{r}-1\right) \sin \theta_{i j}^{r}}\right]^{T}$ and $a_{t}\left(\theta_{i j}^{t}\right)$ $=\frac{1}{\sqrt{N_{t}}}\left[1, e^{j \pi \sin \theta_{i j}^{t}} \ldots e^{j \pi\left(N_{t}-1\right) \sin \theta_{i j}^{t}}\right]^{T}$ denotes the generalised responses of antenna arrays.

A HBF with both analog and digital base-band combiners are used at the receiver side. An analog combiner is denoted by $W_{R F}$ and has the size of $N_{r} \times N_{R F}$. Then the signal is given to the digital base-band combiner denoted by $W_{B}$ of size $N_{R F} \times N_{s}$. Finally, the processed signal is given by equation $(12)$

$$
y=W_{B}^{H} W_{R F}^{H} H V_{R F} V_{B} s+W_{B}^{H} W_{R F}^{H} u
$$

where $N_{r}$ denotes the receive antenna which satisfies the zero-mean Gaussian distribution and covariance matrix of $\sigma^{2} I_{N r}$ which is circularly symmetric. i.e. i.e., $\mathrm{u} \sim C N\left(0, \sigma^{2} I_{N r}\right)$.

\subsection{Problem Formulation}

Let us consider that the Gaussian distribution is followed by the transmitted symbols with the spectral efficiency $\mathrm{R}=\log \operatorname{det}\left(I_{N s}+\frac{1}{\sigma^{2}}\left(\left(W^{H} W\right)^{-1} W^{H}\right) H V V^{H} H^{H} W\right)$. where $\mathrm{V}=V_{R F} V_{B}$ represents the hybrid precoder and $\mathrm{W}=W_{R F} W_{B}$ represents the hybrid combiner. Motivated by [12], [29], [30], to maximize the 
spectral efficiency, the suggested HBF design is connected WMMSE problem in equation (13).

$$
\begin{array}{rrrl}
\underset{V, W, \wedge, \beta}{\operatorname{minimize}} & \operatorname{tr}(\wedge T)-\log \mid & \wedge \mid \\
\text { subject to } & || V \|_{F}^{2} & \leq 1, \\
& \left|\left[\mathrm{~V}_{R F}\right]_{i j}\right|^{2} & =1, \forall \mathrm{i}, \mathrm{j} \\
\text { subject to } & \left|\left[\mathrm{W}_{R F}\right]_{m l}\right|^{2} & =1, \forall \mathrm{m}, 1
\end{array}
$$

where $\beta$ represents the scaling factor, $\wedge$ denotes the weighting matrix of size $N_{s} \times N_{s}$ which is to be optimized and the equation $\mathrm{T} \triangleq \mathrm{E}\left\{\left(\beta^{-1} y-s\right)\left(\beta^{-1} y-\right.\right.$ $\left.s)^{H}\right\}$ represents the updated MSE matrix [9].

\subsection{Design Approach}

The majority of preceding works used gradient based optimization algorithm to optimize the analog precoding matrix. Here, the number of iterations used is proportional to the computational complexity [12]. Hence in this work, a general eigen value decomposition(GEVD) based low complexity algorithm is proposed. In conjunction with similar works [29], [30], the three step design process is ensued to overcome the optimization objective function defined in expression (13) .

\section{Step: 1}

Initially in the first step, $\wedge$ and $V$ is fixed to optimize ' $\mathrm{W}$ ' in (13)

$$
\begin{aligned}
& \underset{W_{R F}, W_{B}}{\operatorname{minimize}} \operatorname{tr}\left(\wedge\left(W^{H} H_{1} H_{1}^{H} W-W^{H} H_{1}-H_{1}^{H} W+\sigma^{2} \beta^{-2} \mathrm{~W}^{H} W+I_{N s}\right)\right) \\
& \quad \text { subject to }\left|\left[\mathrm{W}_{R F}\right]_{m l}\right|=1, \forall \mathrm{m}, \mathrm{l}
\end{aligned}
$$

where $H_{1} \triangleq \mathrm{H} V_{R F} V_{U}$. Then the objective function in (14) is differentiated with respect to $W_{B}$ and the result is equated to zero to get the optimal $W_{B}$. Now, on substituting the optimal $W_{B}$ in (14), the objective function for $W_{R F}$ is given by

$$
I\left(W_{R F}\right) \triangleq \operatorname{tr}\left(\wedge\left(I_{N s}+\sigma^{-2} \beta^{2} H_{1}^{H} W_{R F} \times\left(W_{R F}^{H} W_{R F}\right)^{-1} W_{R F}^{H} H_{1}^{H}\right)^{-1}\right)
$$

\section{Step:2}

Likewise in the second step, using the fixed values of $\mathrm{W}$ and $\mathrm{V}$, the weighting matrix $\wedge$ is further optimized. The objective function defined by expression (13) is differentiated w.r.t. $\wedge$ and is equated to zero. Finally, the optimal weighting matrix $\wedge$ is given by the equation $\wedge=T^{-1}$.

\section{Step:3}

In the final step, using the recently updated values of $\wedge$ and $W$, the optimal value of $\mathrm{V}$ is obtained over the subsequent problem. 


$$
\begin{array}{cc}
\underset{W_{R F}, W_{B}}{\operatorname{minimize}} & \operatorname{tr}\left(\wedge\left(H_{2}^{H} V V^{H} H_{2}-H_{2}^{H} V-V^{H} H_{2}+\sigma^{2} \beta^{-2} \mathrm{~W}^{H} W+I_{N s}\right)\right) \\
\text { subject to } & \|V\|_{F}^{2} \leq 1 \\
& \left|\left[\mathrm{~V}_{R F}\right]_{i j}\right|=1, \forall \mathrm{i}, \mathrm{j}
\end{array}
$$

where $H_{2} \triangleq H^{H} W_{R F} W_{B}$. The optimal $\beta$ is given by $\beta=\left(\operatorname{tr}\left(V_{R F} V_{U} V_{U}^{H} V_{R F}^{H}\right)\right)^{-\frac{1}{2}}$ and the optimal $V_{U}$ is given by $V_{U}=\left(V_{R F}^{H} H_{2} \wedge H_{2}^{H} V_{R F}+\sigma^{2} \psi V_{R F}^{H} V_{R F}\right)^{-1} V_{R F}^{H} H_{2} \wedge$, where during the optimization for $\mathrm{V}, \psi \triangleq \operatorname{tr}\left(\wedge W^{H} W\right)$ is a constant scalar. This optimal $V_{U}$ and $\beta$ are substituted in the objective function in (16), the following equation has been obtained:

$$
J\left(V_{R F}\right) \triangleq \operatorname{tr}\left(\left(\wedge^{-1}+\frac{1}{\sigma^{2} \psi} H_{2}^{H} V_{R F} \times\left(V_{R F}^{H} V_{R F}\right)^{-1} V_{R F}^{H} H_{2}^{H}\right)^{-1}\right)
$$

The above mentioned design steps are performed iteratively in the optimization problem defined by expression (13) via algorithm 1.

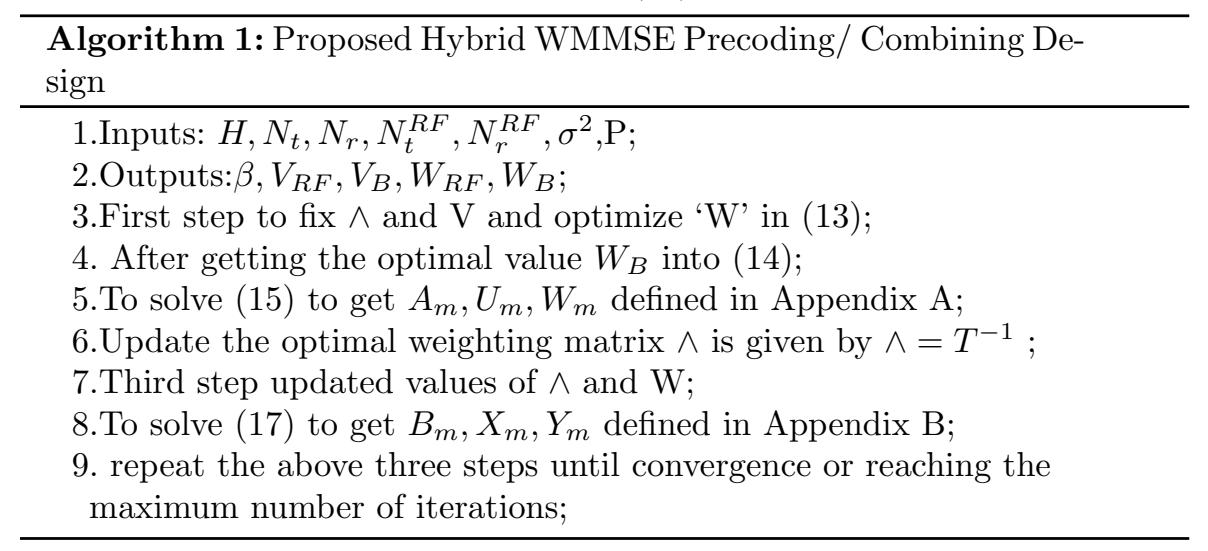

\section{Simulation results}

Based upon the SE performance metrics, the simulation results are furnished to show the effectiveness of the suggested HBF algorithm compared to the analog and fully digital beam-forming algorithms. Simulation parameters are tabulated in Table 4 . An assumption is made that $\alpha_{i j} \sim \mathcal{C} N(0,1)$, based on the Laplacian distribution over mean cluster angles, the angle of arrival and the angle of departure can be estimated and can be uniformly and independently distributed over $[0,2 \pi]$. Here, it is also assumed that the estimation of channel and system synchronization is done perfectly. The transmitter and receiver antennas are fixed to $N_{t}=N_{r}=100$ throughout the entire simulation, except the un-coded modulation scheme Quadrature Phase shift Keying (QPSK) is taken into account. 
Table 4 Simulation Parameters

\begin{tabular}{|l|l|}
\hline Carrier frequency & $60 \mathrm{GHz}$ \\
\hline System Bandwidth & $2 \mathrm{GHz}$ \\
\hline $\begin{array}{l}\text { Number of transmit antenna } \\
\left(N_{t}\right)\end{array}$ & 100 \\
\hline $\begin{array}{l}\text { Number of receive antenna } \\
\left(N_{r}\right)\end{array}$ & 100 \\
\hline Number of clusters $\left(N_{C}\right)$ & 5 \\
\hline Number of Rays $\left(N_{R}\right)$ & 10 \\
\hline Angular Spread & $10^{\circ}$ \\
\hline Modulation Scheme & QPSK \\
\hline Beamforming techniques & OMP, Digital and Hybrid \\
\hline
\end{tabular}

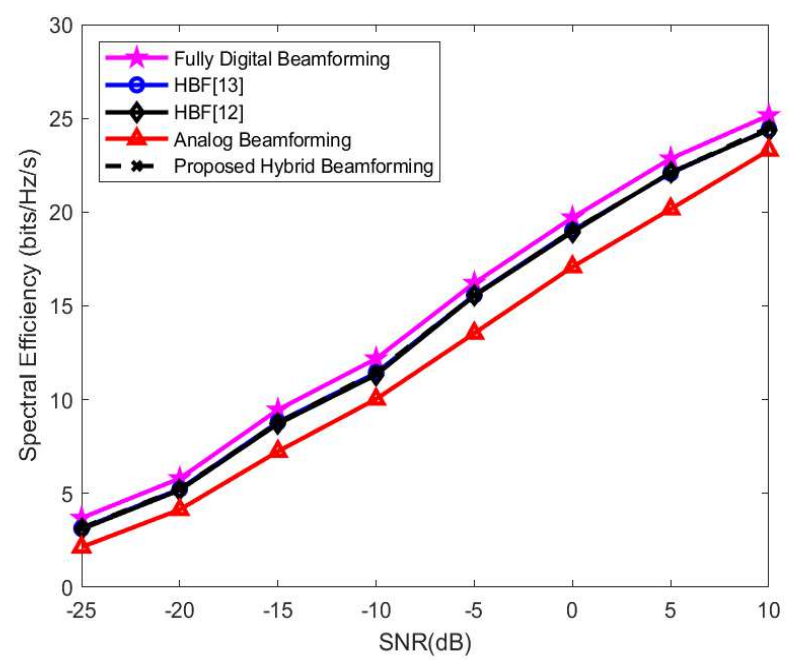

Fig. 9 Spectral efficiencies achieved by the different beamforming algorithm in a $100 \times 100$ mmWave system with $2 \mathrm{RF}$ chains at both the transmitter and receiver.

The spectral efficiencies of different precoding methodologies are shown as a function of SNR with the WMMSE criterion in Fig 9. As SNR(dB) increases, the Spectral Efficiency (bits/Hz/s) increases. From Table 5, it can be concluded that the Digital and Proposed Hybrid Precoding method having high Spectral Efficiency compared to that of the OMP Precoding method at $\mathrm{SNR}=10 \mathrm{~dB}$. The effectiveness of the fully-digital beam-forming algorithm proposed by El Ayach et al. is compared with the traditional analog beam-forming algorithms provided by $\mathrm{Yu} \mathrm{X}$ et al. and Sohrabi and $\mathrm{Yu}$, intending to maximize the spectral efficiency. From the obtained simulation results, it is evident that compared to the OMP algorithms, the performance of other HBF algorithms is quite close. The proposed methodology using WMMSE criterion-based algorithm is effective when compared to conventional HBF algorithms. It is because the 
Table 5 The comparison of different beamforming (BF) algorithms on spectral efficiency $\left(N_{s}=2\right)$

\begin{tabular}{|c|c|c|c|}
\hline Precoding method & No. of Tx Antenna & No. of Rx Antenna & SE (bps/Hz) \\
\hline OMP & 100 & 100 & 23.3 \\
\hline Digital & 100 & 100 & 25.15 \\
\hline Proposed Hybrid & 100 & 100 & 24.52 \\
\hline
\end{tabular}

WMMSE based optimization with suitable weights is another method to meet the objective of increasing the spectral efficiency.

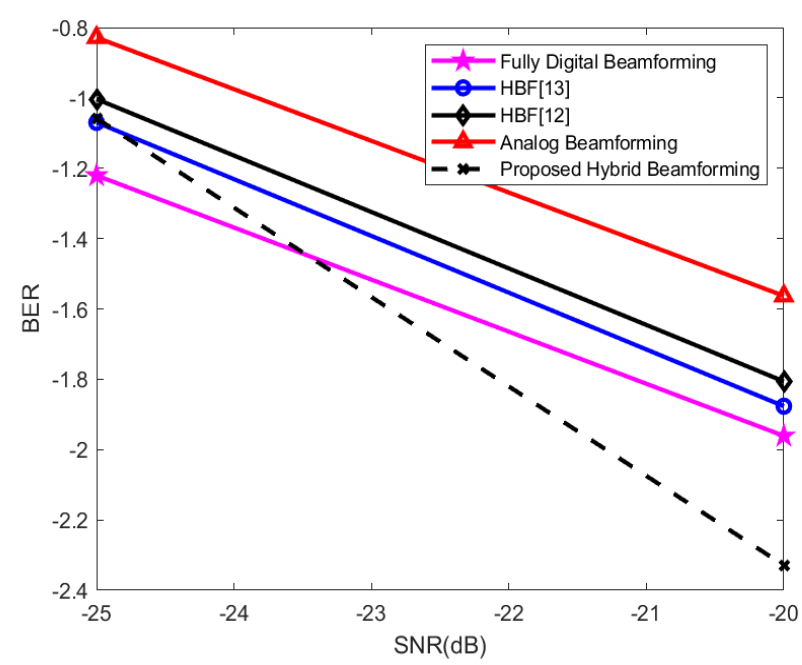

Fig. 10 BER achieved by the different beamforming algorithm in a $100 \times 100 \mathrm{mmWave}$ system with 2 RF chains at both the transmitter and receiver.

The BER performance of different precoding methodologies are shown in fig. 10 as a function of SNR with $N_{s}=N_{R F}=2$. From Fig. 10, it can be observed that, as SNR $(\mathrm{dB})$ increases, the BER (in log scale) decreases Linearly. It is apparent from the results obtained that, relative to the traditional OMPbased methodology, the proposed HBF algorithm substantially outshined and almost achieved full-digital within $1 \mathrm{~dB}$. This is because the OMP based analog beam-forming algorithm is restricted to the pre-defined antenna array response vectors. Therefore, a significant reduction in the size of the feasible set of vectors results in the worst performance amidst all algorithms. From Table 6, it can be concluded that the Bit Error Rate for Proposed Hybrid is lesser compared to Digital and OMP Precoding methods at $\mathrm{SNR}=-20 \mathrm{~dB}$. 
Table 6 The comparison of different beamforming $(\mathrm{BF})$ algorithms having $\operatorname{BER}\left(N_{s}=2\right)$

\begin{tabular}{|c|c|c|c|}
\hline Precoding method & No. of Tx Antenna & No. of Rx Antenna & Bit Error Rate \\
\hline OMP & 100 & 100 & -1.563 \\
\hline Digital & 100 & 100 & -1.961 \\
\hline Proposed Hybrid & 100 & 100 & -2.329 \\
\hline
\end{tabular}

\subsection{Convergence Analysis}

The convergence of the proposed algorithm can be expanded to their counterparts with the WMMSE criterion. The extra optimizing step for the weighting matrix is the key difference in the WMMSE based algorithms. With the help of Karush-Kuhn-Tucker conditions, the solution of the optimal weighting matrix can be found as well ensures the objective function to be minimized. The convergence relies on the analog beamformers' design. Since the weighting matrix is considered a constant matrix in the optimization beamformers' steps, it can be concluded along WMMSE criterion. Unlike the MO-HBF algorithm, the convergence of this algorithm strictly cannot be proved, due to the approximations of $V_{R F}^{H} V_{R F} \approx N_{t} I_{N_{R F}}$ and $W_{R F}^{H} W_{R F} \approx N_{r} I_{N_{R F}}$. The phase extraction operation further raises the difficulty.

\subsection{Complexity Analysis}

The computational complexity for the proposed WMMSE based algorithm is analyzed concerning the no. of complex multiplications since the weighting matrix $(\wedge)$ dimension is $N_{s} \times N_{s}$, and the other additional complexities are trivial. In the proposed GEVD HBF algorithm, the major complexity involves both of the following:

- Preceding the GEVD operation:The complexity involved in the computation of $A_{m}, U_{m}$ and $W_{m}$ is $\left(2 N_{a n t}^{2} N_{R F}+5 N_{R F}^{2} N_{a n t}+2 N_{R F}^{2}+\mathcal{O}\left(N_{R F}^{3}\right)\right)$, where $\mathcal{O}\left(N_{R F}^{3}\right)$ is the complexity of inversion of $N_{R F} \times N_{R F}$ matrix.

- GEVD operation: The order of complexity involved in computing GEVD is $\mathcal{O}\left(N_{R F}^{3}\right)$. The complexity can be mitigated to $\mathcal{O}\left(N_{p} N_{R F}^{2}\right)$ as the computation is performed only on the largest generalized eigenvectors utilizing the power methodology [38]. In the power method, $N_{p}$ is the number of iterations. The simulation is noted that $N_{p}=10$ is highly sufficient to attain an effective result. $N_{\text {out }}\left(\mathcal{O}\left(N_{p} N_{\text {ant }}^{2}\right)+\mathcal{O}\left(N_{R F}^{3}\right)+2 N_{\text {ant }}^{2} N_{R F}+5 N_{R F}^{2} N_{\text {ant }}+\right.$ $2 N_{R F}^{2}$ ) is the final complexity of GEVD Hybrid beamforming.

\subsection{System Level Analysis}

For performance analysis, the proposed Large antenna array with HBF is simulated using the New York University simulator (NYUSIM). It is also used in the deployment of $5 \mathrm{G}$ communication systems [40]. The channel parameters of the simulator, which are based on the proposed link budget and antenna 
Table 7 Input parameters for NYUSIM Simulator

\begin{tabular}{|c|c|}
\hline Parameters & Value \\
\hline Frequency $(\mathrm{GHz})$ & 60 \\
\hline RF bandwidth(MHz) & 800 \\
\hline Scenario & UMi \\
\hline Environment & LOS \\
\hline Lower Bound of T-R Separation Distance $(\mathrm{m})$ & 10 \\
\hline Upper Bound of T-R Separation Distance $(\mathrm{m})$ & 200 \\
\hline TX Power $(\mathrm{dBm})$ & 30 \\
\hline Base Station Height $(\mathrm{m})$ & 35 \\
\hline User Terminal Height(m) & 1.5 \\
\hline No. of Rx Locations & 1 \\
\hline Barometric Pressure (mbar) & 1013.25 \\
\hline Humidity $(\%)$ & 50 \\
\hline Temperature $\left({ }^{\circ} \mathrm{C}\right)$ & 20 \\
\hline Rain Rate(mm/hr) & 0 \\
\hline Polarization & Co-Pol \\
\hline Foliage Loss & No \\
\hline Distance within Foliage $(\mathrm{m})$ & 0 \\
\hline Foliage Attenuation $(\mathrm{dB} / \mathrm{m})$ & 0.4 \\
\hline Outdoor to Indoor(O2I) Penetration Loss & No \\
\hline O2I Loss Type & Low Loss \\
\hline TX Array Type & ULA \\
\hline RX Array Type & ULA \\
\hline No. of TX Antenna Elements $\left(N_{t}\right)$ & 100 \\
\hline No. of RX Antenna Elements $\left(N_{r}\right)$ & 100 \\
\hline TX Antenna Spacing(in wavelength) & 0.5 \\
\hline RX Antenna Spacing(in wavelength) & 0.5 \\
\hline No. of TX Antenna Elements Per Row $W_{t}$ & 1 \\
\hline No. of RX Antenna Elements Per Row $W_{r}$ & 1 \\
\hline TX Antenna Azimuth HPBW & $10^{\circ}$ \\
\hline TX Antenna Elevation HPBW & $10^{\circ}$ \\
\hline RX Antenna Azimuth HPBW & $10^{\circ}$ \\
\hline RX Antenna Elevation HPBW & $10^{\circ}$ \\
\hline
\end{tabular}

properties, are specified as per the large antenna array design on the graphical user interface (GUI), as illustrated in Table 7. For the channel propagation, the panel channel parameters comprise 16 primary input attributes, and for the antenna array transmission and reception, the panel Antenna properties contain 12 input attributes. Fig. 11 \& 12 demonstrate the power spectrum of simulated AOA and AOD with its respective omnidirectional and directional Power Delay Profile (PDP), which is depicted in Fig. 13 \& 14. Fig. 15 shows the small-scale PDP of the Tx-Rx separation distance of $164.8 \mathrm{~m}$ at frequency $60 \mathrm{GHz}$ for indoor deployments.

Fig. 16 shows the scattered plot of path loss precipitated after the ' 100 ' number of uninterrupted simulation runs, displaying the directional and omnidirectional path loss values of the whole distance span produced from the ' 100 ' number of uninterrupted simulation runs. In addition to the fitted PLE and shadow fading standard deviation employing MMSE methodology [41],[42]. In 
Fig. 16, the legend inside the figure represents ' $n$ ' for PLE, $\sigma$ is the shadow fading standard deviation, "Omni" represents omnidirectional, "dir" denotes directional, and the "dir-best" represents the more vital power reception direction. To produce the directional path loss for every receiver location, NYUSIM helps to search almost all possible pointing angles with incremental azimuth and the user-specified antenna for transmission or reception elevation HPBWs GUI after the generation of omnidirectional PDP. Total directional path loss equals the power at the transmitter and the transmitter, and the receiver antenna gains, subtracting the received directional power [2], [44]. The simulated PLE and shadow fading standard deviation values achieved in the directional power delay profile are $\mathrm{PLE}=2.7, \mathrm{PL}=128.7 \mathrm{~dB}$, and $P_{r}=49.4 \mathrm{dBm}$. As most of the multi-path components are filtered out spatially, the directional PLE and the directional path loss values are significantly more prominent when compared to the omnidirectional values because of their directional pattern. Thereby the receiver receives only a few multi-path components; therefore, it has reduced energy consumption, and hence it has higher directional pathloss after the removal of the effect of antenna gain from the received power $[42],[43],[44]$. The directional and the omnidirectional antenna array that gains during the transmission and reception is almost equal to the proposed large antenna array gain in section III.

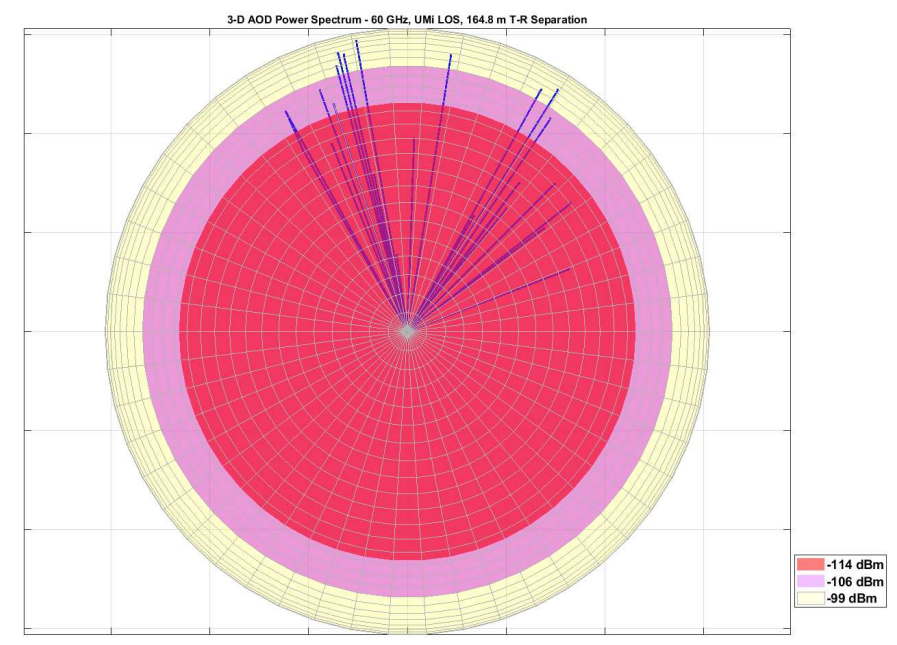

Fig. 11 Simulated 3D-AOD power spectrum. 


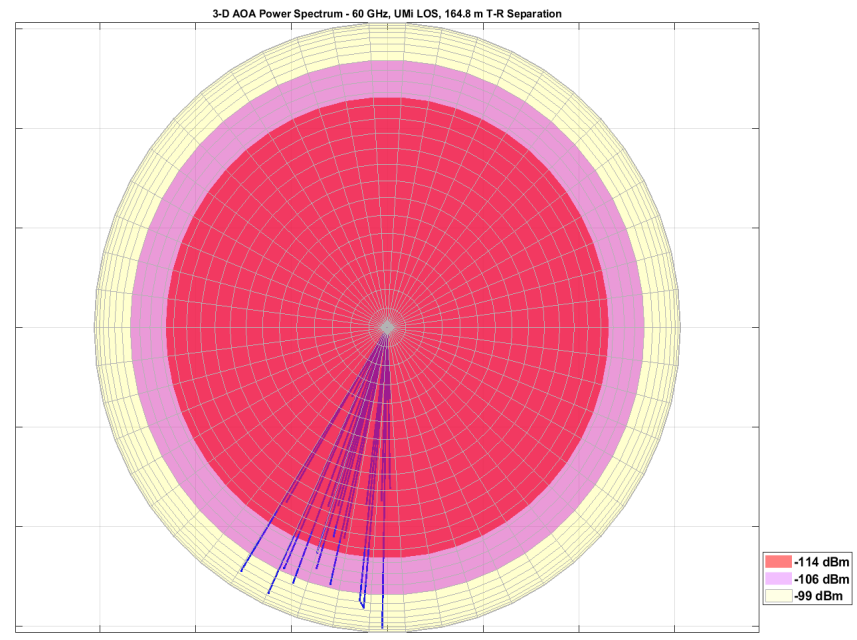

Fig. 12 Simulated 3D-AOA power spectrum.

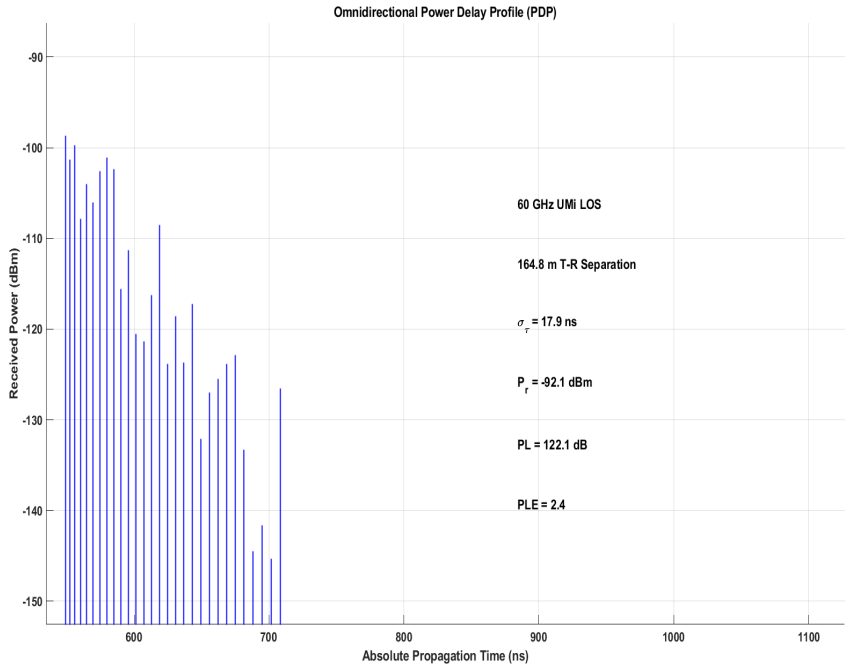

Fig. 13 Simulated omnidirectional Power Delay Profile.

\subsection{System Capacity Analysis}

The proposed system operates at the bandwidth of $2 \mathrm{GHz}$ Time Division Duplex (TDD) mode, where $1 \mathrm{GHz}$ of Bandwidth is shared by each uplink and downlink $(1 \mathrm{GHz}+1 \mathrm{GHz})$ to test the large antenna array system functionality using beam-forming techniques. A standardized distribution of transmitter $(\mathrm{Tx})$ cell sites is rendered in a hexagonal pattern, consisting of three cells per 


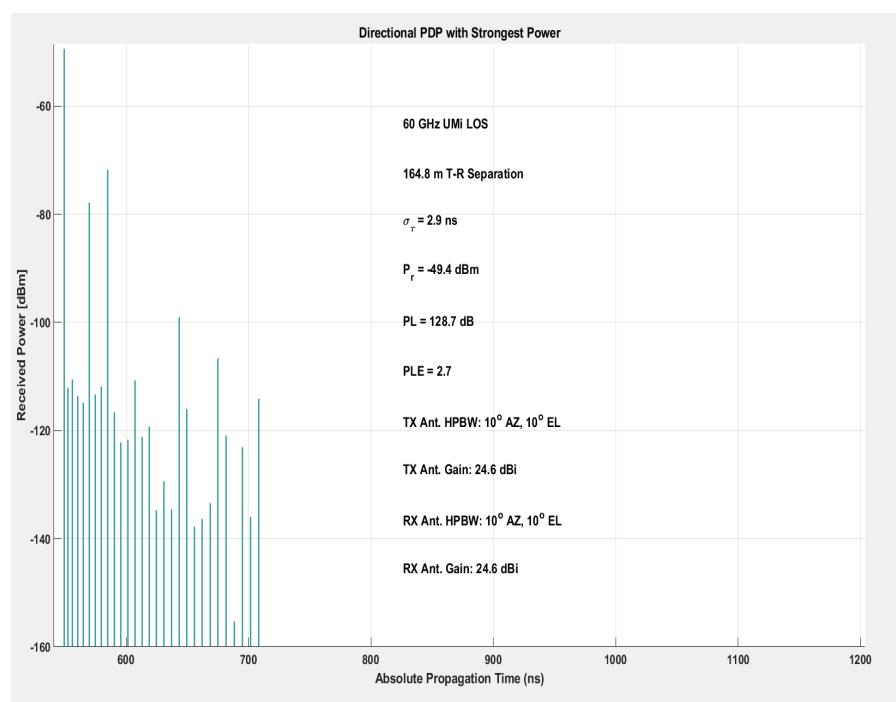

Fig. 14 Simulated Directional Power Delay Profile with Strongest Power.

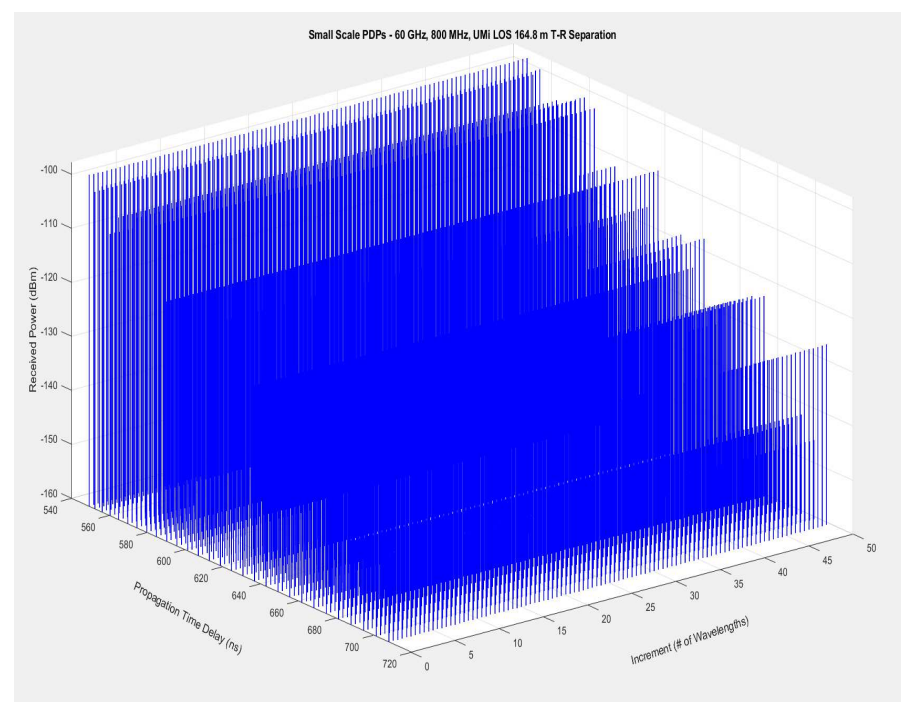

Fig. 15 Small Scale power delay profile at $60 \mathrm{GHz}$ RF Bandwidth of $800 \mathrm{MHz}$.

site covering $2 \mathrm{~km}$ by $2 \mathrm{~km}$ with 200 meters of ISD, like a 3GPP UMi model (around 390 cells). Similarly, in an environment of just one consumer per cell, a uniform distribution of recipients $(\mathrm{Rx})$ is made, which also parallels the assumption made in the 3GPP UMi model [29]. Area-throughput (C) is a highly indispensable efficiency parameter for new-future cellular networks, calculated in $b i t / s / \mathrm{km}^{2}$ and modeled using the formula provided by the expression (18):

$$
C=B \times D \times S E
$$




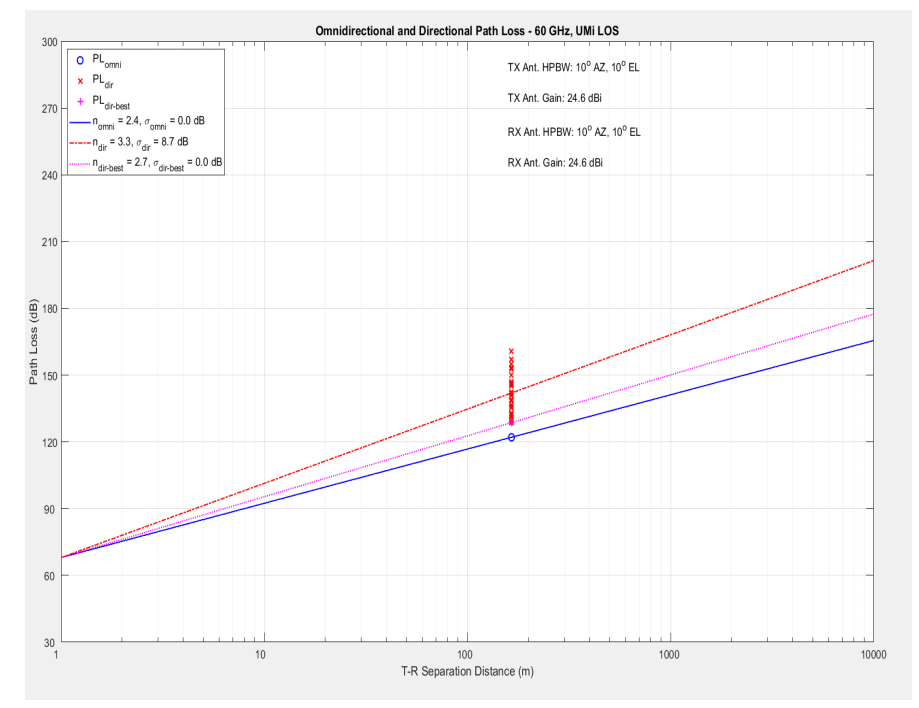

Fig. 16 The directional and omni-directional path loss with 100 simulation runs

where $\mathrm{B}$ is bandwidth, D is the average cell density, and $\mathrm{SE}$ is the spectral efficiency per cell.

Table 8 Throughput analysis of different beamforming methodologies

\begin{tabular}{|c|c|c|c|c|c|}
\hline System & $\begin{array}{c}\text { System } \\
\text { Bandwidth } \\
(\mathrm{GHz})\end{array}$ & $\begin{array}{c}\text { No.of Antenna } \\
(\mathrm{Tx}-\mathrm{Rx})\end{array}$ & $\begin{array}{c}\text { Type of } \\
\text { Beamforming }\end{array}$ & $\begin{array}{c}\text { System } \\
\text { throughput } \\
(\mathrm{Gbps} / \mathrm{cell})\end{array}$ & $\begin{array}{c}\text { Area } \\
\text { throughput } \\
\left(\mathrm{Gbps} / \mathrm{km}^{2}\right)\end{array}$ \\
\hline mmWave & $1+1$ & 100 & OMP & 23.3 & 9087 \\
\hline mmWave & $1+1$ & 100 & Digital & 25.15 & 9808 \\
\hline mmWave & $1+1$ & 100 & Hybrid & 24.52 & 9563 \\
\hline
\end{tabular}

Using these presumptions, the proposed mmWave system employing a 60 $\mathrm{GHz}$ band 100-element ULA generates a significant 1000-fold enhancement in throughput compared to the Long Term Evolution (LTE) systems. It is advantageous to have a distended spectrum: mmWave is selected with the bandwidth of $2 \mathrm{GHz}$, by $20+20 \mathrm{MHz}(40 \mathrm{MHz})$ of LTE Duplex, with 100 times the bandwidth of mmWave system. However, the target of link budget is to attain $2 \mathrm{GHz}$ bandwidth with a data of $11.3 \mathrm{Gbps}$. And by employing the proposed large antenna array with HBF technique, the system throughput of $24.52 \mathrm{Gbps} / \mathrm{cell}$ is attained. The comparison of system throughput and area throughput for various beamforming techniques is shown in Table 8 .

\section{Conclusion}

The $60 \mathrm{GHz}$ mmWave communications are highly suitable for 5G indoor point-to-point communications. Additionally, for a slow-moving user equip- 
ment, mmWave communication provides high throughput . Here, the HBF technique is employed which needs only a tiny number of RF chains and a large antenna array for transmission and reception. This research further verifies that by integrating a large antenna array, beamforming techniques and mmWave frequencies results in improved spectral efficiency of $24.52 \mathrm{bps} / \mathrm{Hz}$. It can be concluded that the overall throughput of the $\mathrm{HBF}$ is relative to digital beamforming techniques and outperforms the OMP beamforming technique. Therefore, the proposed large antenna array with HBF technique is a very efficient solution for network deployment of small cell $5 \mathrm{G}$ indoor point-to-point communications.

\section{Appendix A - Proof of the equation(15):}

In accordance with [13], an approximation is made for large scale MIMO systems that $W_{R F}^{H} W_{R F} \approx N_{r} I_{N_{R F}}$, this approximation, the equation (15) can be simplified as follows:

$$
I\left(W_{R F}\right) \approx \operatorname{tr}\left(\wedge\left(I_{N s}+\frac{\sigma^{-2} \beta^{2}}{N_{r}} H_{1}^{H} W_{R F} W_{R F}^{H} H_{1}^{H}\right)^{-1}\right)
$$

With this simplified form, an optimal value of $W_{R F}$ can be obtained on columnby-column basis. Particularly, $\bar{W}_{m}$ is defined as the left-out sub-matrix of $W_{R F}$ after taking out the $m^{t h}$ column $w_{m}$. Furthermore, define $A_{m} \triangleq I_{N s}+$ $\frac{\sigma^{-2} \beta^{2}}{N_{r}} H_{1}^{H} \bar{W}_{m} \bar{W}_{m}^{H} H_{1}^{H}$. Then, using $(A+B)^{-1}=A^{-1}-\frac{A^{-1} B A^{-1}}{1+\operatorname{tr}\left(A^{-1} B\right)}$ in (19), it can be written as follows:

$$
\begin{aligned}
I\left(W_{R F}\right) \approx \operatorname{tr}\left(\wedge A_{m}^{-1}\right)- & \wedge \times \frac{\operatorname{tr}\left(\frac{\sigma^{-2} \beta^{2}}{N_{r}} A_{m}^{-1} H_{1}^{H} w_{m} w_{m}^{H} H_{1}^{H} A_{m}^{-1}\right)}{1+\operatorname{tr}\left(\frac{\sigma^{-2} \beta^{2}}{N_{r}} A_{m}^{-1} H_{1}^{H} w_{m} w_{m}^{H} H_{1}^{H}\right)} \\
& =\operatorname{tr}\left(\wedge A_{m}^{-1}\right)-\wedge \times \frac{w_{m}^{H} U_{m} w_{m}}{w_{m}^{H} W_{m} w_{m}}
\end{aligned}
$$

where $U_{m}=\frac{\sigma^{-2} \beta^{2}}{N_{r}} H_{1}^{H} A_{m}^{-2} H_{1}^{H}$ and $W_{m}=\frac{1}{N_{r}} I_{N_{r}}+\frac{\sigma^{-2} \beta^{2}}{N_{r}} H_{1}^{H} A_{m}^{-1} H_{1}^{H}$ are both Hermitian matrices.

\section{Appendix B - Proof of the equation(17):}

Following Appendix A, solving equation (17) in a similar way, we get

$$
J\left(V_{R F}\right) \approx \operatorname{tr}\left(\left(\wedge^{-1}+\frac{1}{\sigma^{2} \psi N_{t}} H_{2}^{H} V_{R F} V_{R F}^{H} H_{2}^{H}\right)^{-1}\right)
$$

Further define $B_{m} \triangleq\left(\wedge^{-1}+\frac{1}{\sigma^{2} \psi N_{t}} H_{2}^{H} \overline{V_{m}} \overline{V_{m}^{H}} H_{2}^{H}\right)$. Then, using $(A+B)^{-1}=$ $A^{-1}-\frac{A^{-1} B A^{-1}}{1+\operatorname{tr}\left(A^{-1} B\right)}$ the expression in $(21)$ can also be simplified as follows: 


$$
\begin{array}{r}
J\left(V_{R F}\right) \approx \operatorname{tr}\left(\wedge B_{m}^{-1}\right)+\frac{\operatorname{tr}\left(\frac{1}{\sigma^{2} \psi N_{t}} \wedge B_{m}^{-1} H_{2}^{H} v_{m} v_{m}^{H} H_{2}^{H} \wedge B_{m}^{-1}\right)}{1+\operatorname{tr}\left(\frac{1}{\sigma^{2} \psi N_{t}} \wedge B_{m}^{-1} H_{2}^{H} V_{m} V_{m}^{H} H_{2}^{H}\right)} \\
=\operatorname{tr}\left(\wedge B_{m}^{-1}\right)-\frac{v_{m}^{H} X_{m} v_{m}}{v_{m}^{H} Y_{m} v_{m}}
\end{array}
$$

where $X_{m}=\frac{1}{\sigma^{2} \psi N_{t}} H_{2}^{H} \wedge^{2} B_{m}^{-2} H_{2}^{H}$ and $Y_{m}=\frac{1}{N_{t}} I_{N_{t}}+\frac{1}{\sigma^{2} \psi N_{t}} H_{2}^{H} \wedge B_{m}^{-1} H_{2}^{H}$ are both Hermitian matrices.

\section{Acknowledgment}

The authors sincerely acknowledge and would like to thank Shihao Ju, Shu Sun, and Theodore. S. Rappaport for providing NYUSIM Software for 5G System Level Simulation Analysis.

\section{Conflict of Interest:}

The authors declare that they have no conflict of interest

\section{References}

1. Pi, Z. and F. Khan. 2011. "An introduction to millimeter-wave mobile broadband systems". IEEE Commun. Mag. 49(6): 101- 107.

2. Rappaport, T. S., S. Sun, R. Mayzus, H. Zhao, Y. Azar, K. Wang, G. N. Wong, J. K. Schulz, M. Samimi, and F. Gutierrez. 2013. "Millimeter wave mobile communications for 5G cellular: It will work!" IEEE Access. 1: 335-349.

3. Uwaechia, A. N., Mahyuddin, N. M. (2020). A comprehensive survey on millimeter wave communications for fifth-generation wireless networks: feasibility and challenges. IEEE Access, 8, 62367-62414.

4. Busari, S. A., Huq, K. M. S., Mumtaz, S., Dai, L., Rodriguez, J. (2017). Millimeter-wave massive MIMO communication for future wireless systems: A survey. IEEE Communications Surveys Tutorials, 20(2), 836-869.

5. Friis, H. T. 1946. "A note on a simple transmission formula". Proc. IRE. 34(5): 254-256.

6. Yan, H., Ramesh, S., Gallagher, T., Ling, C., Cabric, D. (2019). Performance, power, and area design trade-offs in millimeter-wave transmitter beamforming architectures. IEEE Circuits and Systems Magazine, 19(2), 33-58.

7. Rappaport, T. S., R. W. Heath Jr, R. C. Daniels, and J. N. Murdock. 2014. Millimeter wave wireless communications. Pearson Education.

8. Jeyakumar, P., Malar, E., Idnani, N., Muthuchidambaranathan, P. (2021). Large Antenna Array with Hybrid Beamforming System for 5G Outdoor Mobile Broadband Communication Deployments. Wireless Personal Communications, 1-27.

9. Xiao, M., S. Mumtaz, Y. Huang, L. Dai, Y. Li, M. Matthaiou, G. K. Karagiannidis, E. Björnson, K. Yang, C.-L. I, and A. Ghosh. 2017. "Millimeter Wave Communications for Future Mobile Networks". IEEE J. Sel. Areas Commun. 35(9): 1909- 1935.

10. Alkhateeb, A., O. El Ayach, G. Leus, and R. W. Heath. 2013. "Hybrid precoding for millimeter wave cellular systems with partial channel knowledge". In: Proc. IEEE ITA. IEEE. 1-5.

11. Mo, J. and R. W. Heath. 2014. "High SNR capacity of millimeter wave MIMO systems with one-bit quantization". In: Proc. IEEE ITA. IEEE. 1-5. 
12. Lin, T., Cong, J., Zhu, Y., Zhang, J., Letaief, K. B. (2019). Hybrid beamforming for millimeter wave systems using the MMSE criterion. IEEE Transactions on Communications, 67(5), 3693-3708.

13. Tsang, Y. M., Poon, A. S., Addepalli, S. (2011, December). Coding the beams: Improving beamforming training in mmwave communication system. In 2011 IEEE Global Telecommunications Conference-GLOBECOM 2011 (pp. 1-6). IEEE.

14. Wang, J., Lan, Z., Pyo, C. W., Baykas, T., Sum, C. S., Rahman, M. A., ... Kato, S. (2009). Beam codebook based beamforming protocol for multi-Gbps millimeter-wave WPAN systems. IEEE Journal on Selected Areas in Communications, 27(8), 1390-1399.

15. IEEE Computer Society LAN MAN Standards Committee. (1999). Wireless LAN medium access control (MAC) and physical layer (PHY) specifications. ANSI/IEEE Std. 802.11-1999.

16. El Ayach, O., Heath, R. W., Abu-Surra, S., Rajagopal, S., Pi, Z. (2012, June). Low complexity precoding for large millimeter wave MIMO systems. In 2012 IEEE international conference on communications (ICC) (pp. 3724-3729). IEEE

17. Zhang, X., Molisch, A. F., Kung, S. Y. (2005). Variable-phase-shift-based RF-baseband codesign for MIMO antenna selection. IEEE Transactions on Signal Processing, 53(11), 4091-4103.

18. Venkateswaran, V., van der Veen, A. J. (2010). Analog beamforming in MIMO communications with phase shift networks and online channel estimation. IEEE Transactions on Signal Processing, 58(8), 4131-4143.

19. Bogale, T. E., Le, L. B. (2014, December). Beamforming for multiuser massive MIMO systems: Digital versus hybrid analog-digital. In 2014 IEEE Global Communications Conference (pp. 4066-4071). IEEE.

20. O. El Ayach, S. Rajagopal, S. Abu-Surra, Z. Pi, and R. W. Heath, Jr., "Spatially sparse precoding in millimeter wave MIMO systems," IEEE Trans. Wireless Commun., vol. 13, no. 3, pp. 1499-1513, Mar. 2014.

21. M. Kim and Y. H. Lee, "MSE-based hybrid RF/baseband processing for millimeter-wave communication systems in MIMO interference channels," IEEE Trans. Veh. Technol., vol. 64, no. 6, pp. 2714-2720, Jun. 2015.

22. X. Yu, J.-C. Shen, J. Zhang, and K. B. Letaief, "Alternating minimization algorithms for hybrid precoding in millimeter wave MIMO systems," IEEE J. Sel. Topics Signal Process., vol. 10, no. 3, pp. 485-500, Apr. 2016.

23. F. Sohrabi and W. Yu, "Hybrid digital and analog beamforming design for large-scale antenna arrays," IEEE J. Sel. Topics Signal Process., vol. 10, no. 3, pp. 501-513, Apr. 2016.

24. R. Mai, D. H. N. Nguyen, and T. Le-Ngoc, "MMSE hybrid precoder design for millimeter-wave massive MIMO systems," in Proc. IEEE Wireless Commun. Netw. Conf., Doha, Qatar, Apr. 2016, pp. 1-6.

25. H. Sampath, P. Stoica, and A. Paulraj, "Generalized linear precoder and decoder design for MIMO channels using the weighted MMSE criterion," IEEE Trans. Commun., vol. 49, no. 12, pp. 2198-2206, Dec. 2001.

26. D. P. Palomar, J. M. Cioffi, and M. A. Lagunas, "Joint Tx-Rx beamforming design for multicarrier MIMO channels: A unified framework for convex optimization," IEEE Trans. Signal Process., vol. 51, no. 9, pp. 2381-2401, Sep. 2003.

27. S. Shi, M. Schubert, and H. Boche, "Downlink MMSE transceiver optimization for multiuser MIMO systems: Duality and sum-MSE minimization," IEEE Trans. Signal Process., vol. 55, no. 11, pp. 5436-5446, Nov. 2007.

28. M. Joham, W. Utschick, and J. A. Nossek, "Linear transmit processing in MIMO communications systems," IEEE Trans. Signal Process., vol. 53, no. 8, pp. 2700-2712, Aug. 2005.

29. S. S. Christensen, R. Agarwal, E. De Carvalho, and J. M. Cioffi, "Weighted sum-rate maximization using weighted MMSE for MIMOBC beamforming design," IEEE Trans. Wireless Commun., vol. 7, no. 12, pp. 4792-4799, Dec. 2008.

30. D. H. N. Nguyen, L. B. Le, and T. Le-Ngoc, "Hybrid MMSE precoding for mmWave multiuser MIMO systems," in Proc. IEEE Int. Conf. Commun. (ICC), May 2016, pp. 1-6. 
31. D. H. Nguyen, L. B. Le, T. Le-Ngoc, and R. W. Heath, Jr., "Hybrid MMSE precoding and combining designs for mmWave multiuser systems," IEEE Access, vol. 5, pp. 19167-19181, 2017.

32. J. Joung and Y. H. Lee, "Regularized channel diagonalization for multiuser MIMO downlink using a modified MMSE criterion," IEEE Trans. Signal Process., vol. 55, no. 4, pp. 1573-1579, Apr. 2007.

33. V. Stankovic and M. Haardt, "Generalized design of multi-user MIMO precoding matrices," IEEE Trans. Wireless Commun., vol. 7, no. 3, pp. 953-961, Mar. 2008.

34. Hemadeh, I. A., Satyanarayana, K., El-Hajjar, M., Hanzo, L. (2017). Millimeter-wave communications: Physical channel models, design considerations, antenna constructions, and link-budget. IEEE Communications Surveys Tutorials, 20(2), 870-913.

35. T. M. Cover and J. A. Thomas, Elements of Information Theory, 2nd ed.Hoboken, NJ, USA: Wiley, 2006.

36. C. A. Balanis, Antenna Theory: Analysis and Design, 3rd ed. Hoboken, NJ, USA: Wiley, 2005.

37. ETSI EN 302 217-4- V2.1 (2017-05) -Fixed Radio Systems; Characteris- tics and requirements for point-to-point equipment and antennas; Part 4: An- tennas, 2017.

38. W. Kozlowski, "The power method for the generalized eigenvalue problem," Roczniki Polskiego Towarzystwa Matematycznego, Ser. III, Matematyka Stosowana, Appl. Math., vol. 35, pp. 21-32, 1992.

39. Akdeniz, M. R., Liu, Y., Samimi, M. K., Sun, S., Rangan, S., Rappaport, T. S., Erkip, E. (2014). Millimeter wave channel modeling and cellular capacity evaluation. IEEE journal on selected areas in communications, 32(6), 1164-1179.

40. Sun, S., MacCartney, G. R., Rappaport, T. S. (2017, May). A novel millimeter-wave channel simulator and applications for $5 \mathrm{G}$ wireless communications. In 2017 IEEE International Conference on Communications (ICC) (pp. 1-7). IEEE.

41. S. Sun et al., "Investigation of prediction accuracy, sensitivity, and parameter stability of large-scale propagation path loss models for 5G wireless communications," IEEE Transactions on Vehicular Technology, vol. 65, no. 5, pp. 2843-2860, May 2016.

42. G. R. MacCartney, Jr. et al., "Indoor office wideband millimeter-wave propagation measurements and channel models at 28 and $73 \mathrm{GHz}$ for ultra-dense 5G wireless networks," IEEE Access, vol. 3, pp. 2388-2424, Oct. 2015.

43. M. K. Samimi and T. S. Rappaport, "3-D millimeter-wave statistical channel model for 5G wireless system design," IEEE Transactions on Microwave Theory and Techniques, vol. 64, no. 7, pp. 2207-2225, July 2016.

44. T. S. Rappaport, G. R. MacCartney, Jr., M. K. Samimi, and S. Sun, "Wideband millimeter-wave propagation measurements and channel models for future wireless communication system design (Invited Paper)," IEEE Transactions on Communications, vol. 63 , no. 9, pp. 3029-3056, Sep. 2015. 
Figures

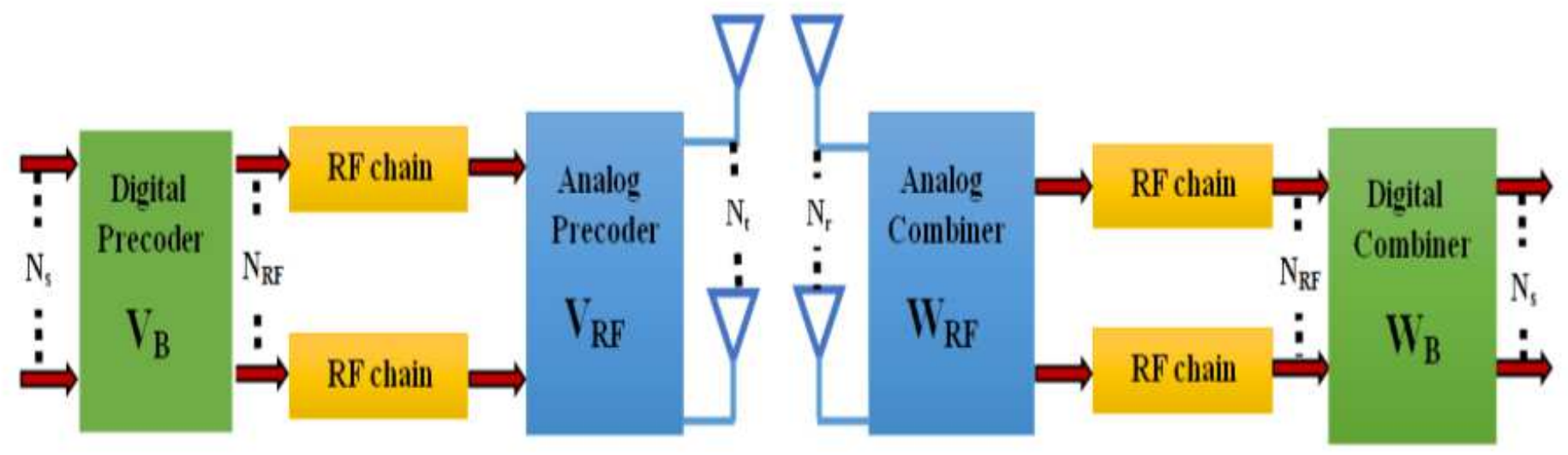

Figure 1

Point-to-Point mmWave Communication

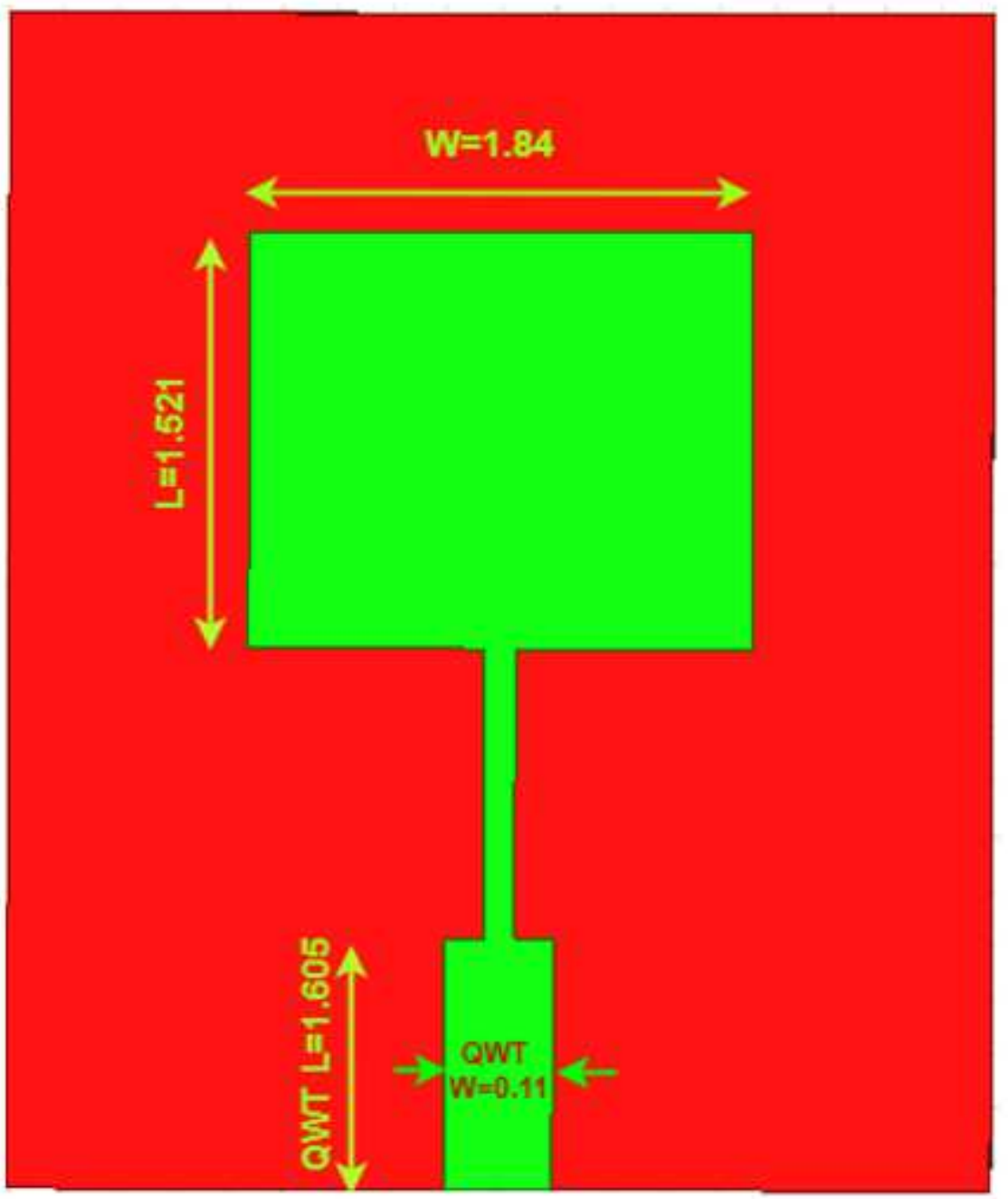

Figure 2 
Single Microstrip Patch antenna

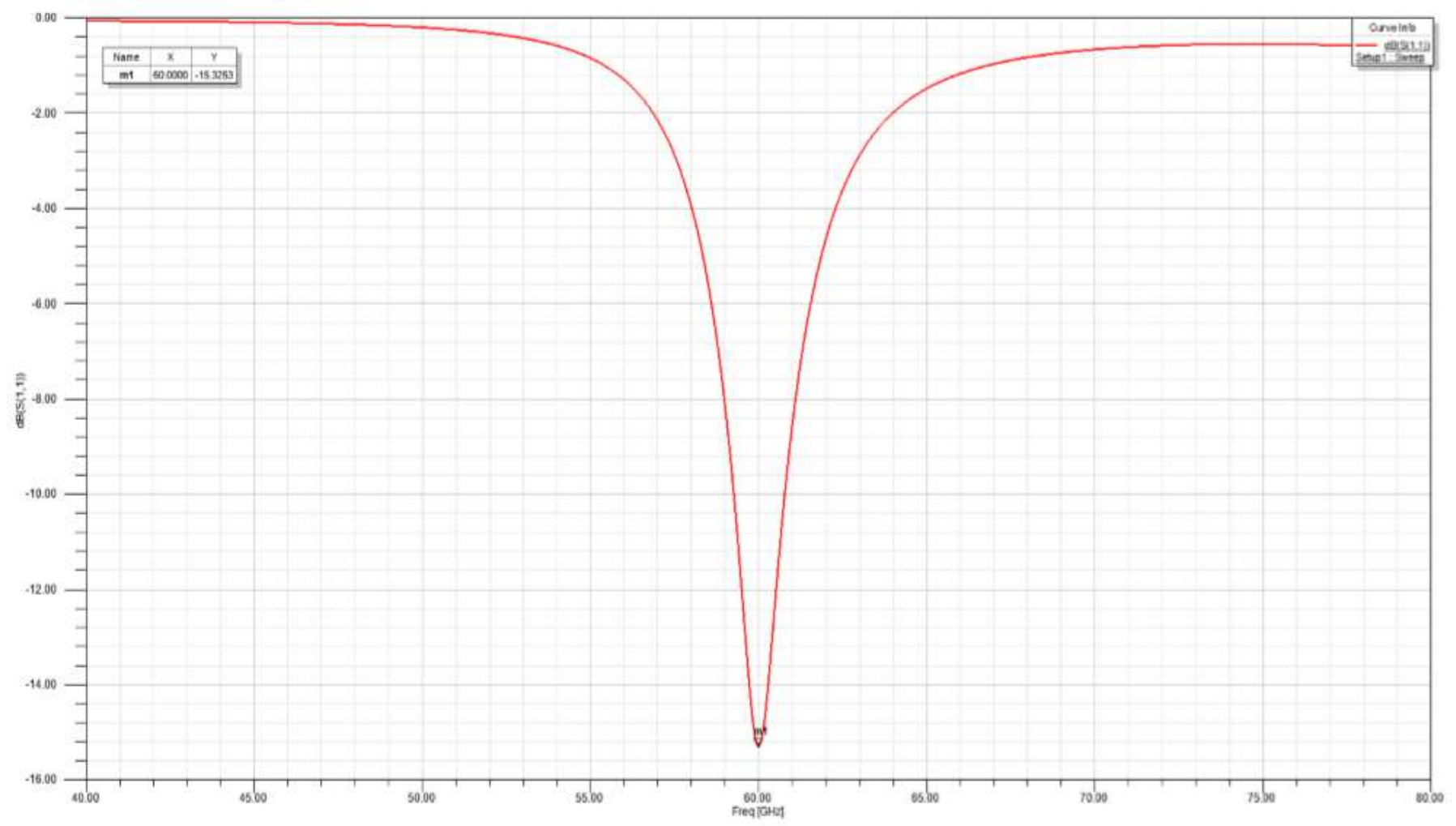

Figure 3

Return loss graph of the single patch antenna at $60 \mathrm{GHz}$ carrier frequency 


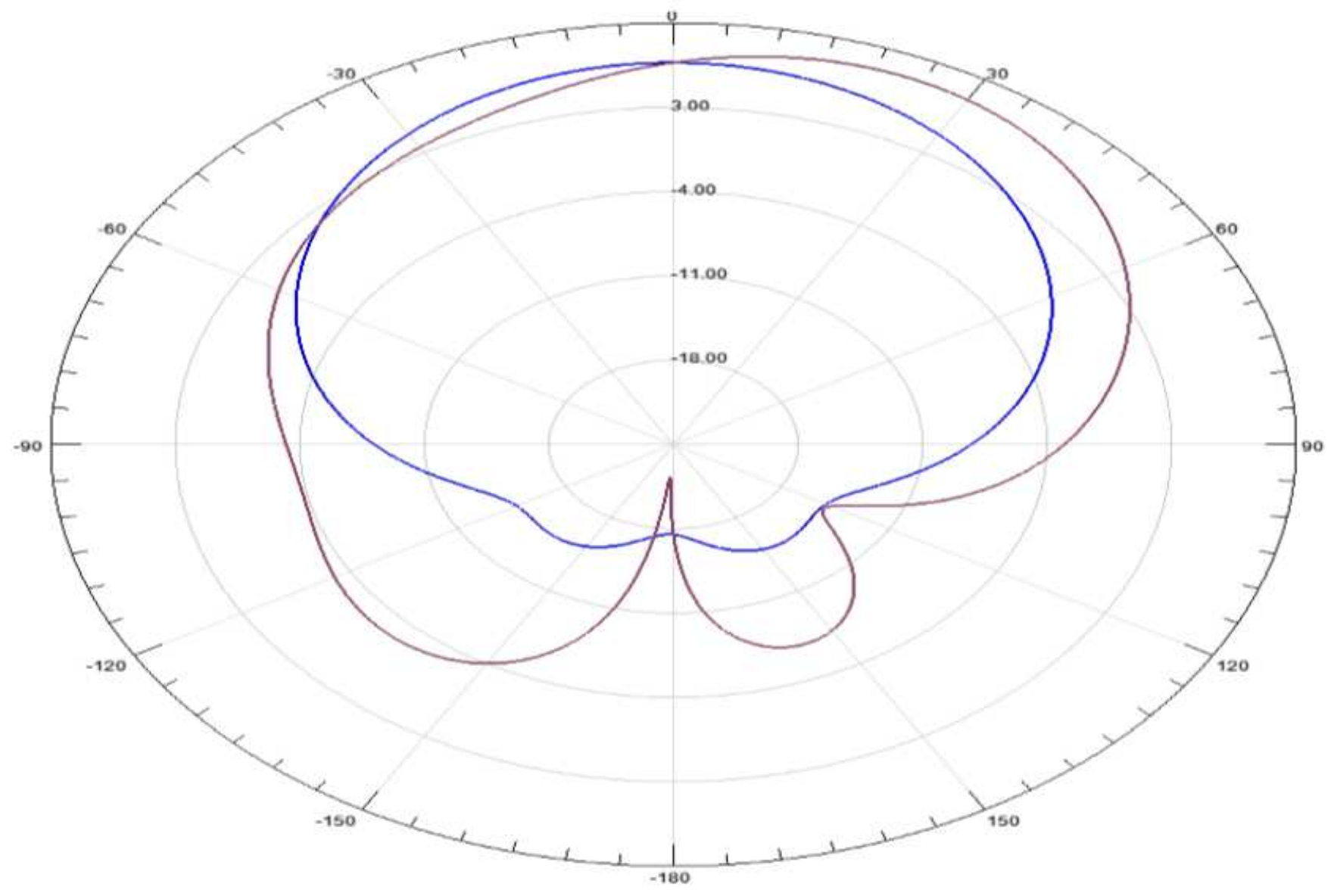

Figure 4

Radiation pattern of the single patch antenna for $\mathrm{H}$-plane and E-plane 


\section{dB (GainTota1)}

$8.1907 e+000$

6. $2851 e+000$

4. $3796 e+000$

2. $4741 e+000$

5. $6856 e-001$

$-1.3970 e+000$

$-3.2425 e+000$

$-5.1480 e+000$

$-7.0535 e+000$

$-8.9591 e+000$

$-1.0865 e+001$

$-1.2770 e+001$

$-1.4676 e+001$

$-1.6581 e+\square 01$

$-1.8487 e+001$

$-2.0392 e+001$

$-2.2298 e+001$

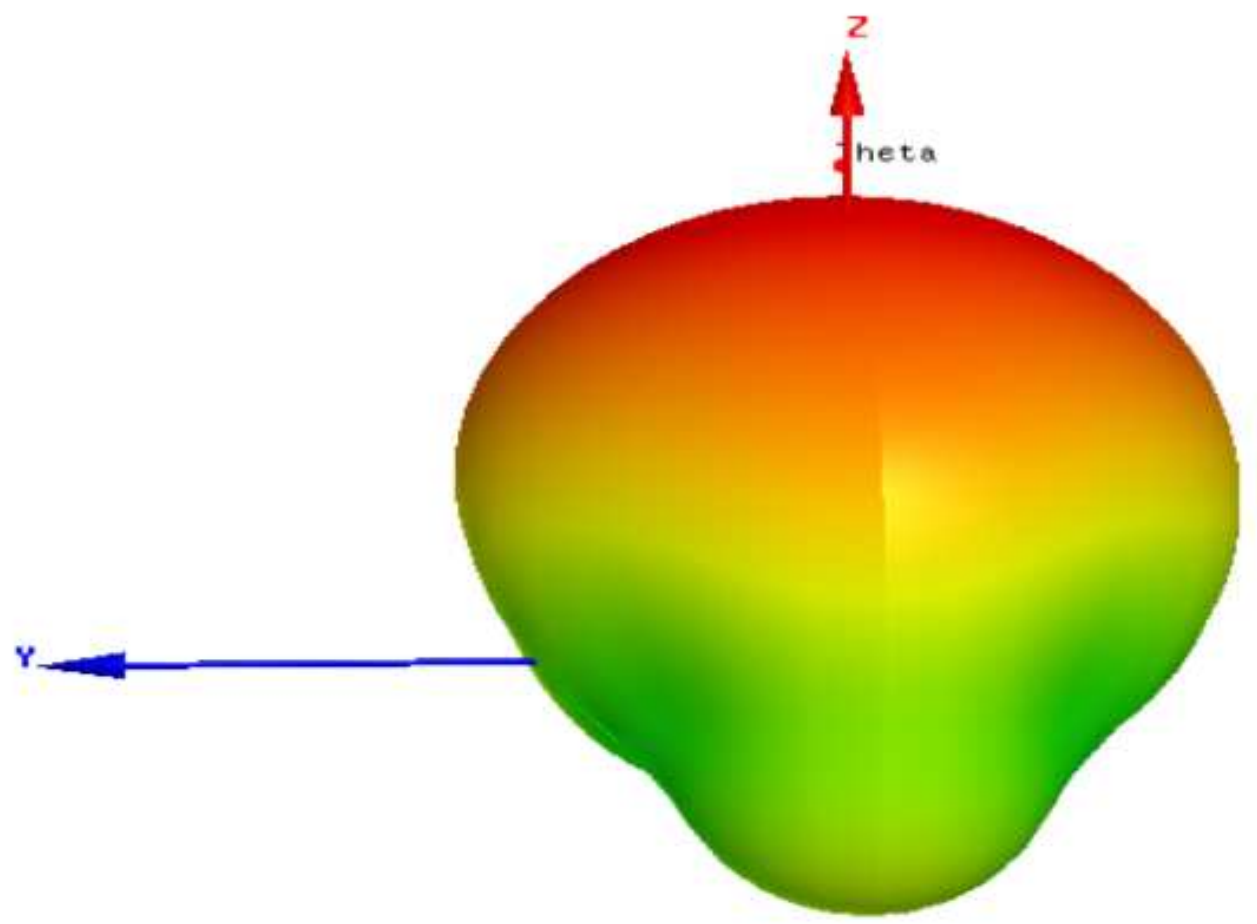

Figure 5

Gain response of the single element antenna 


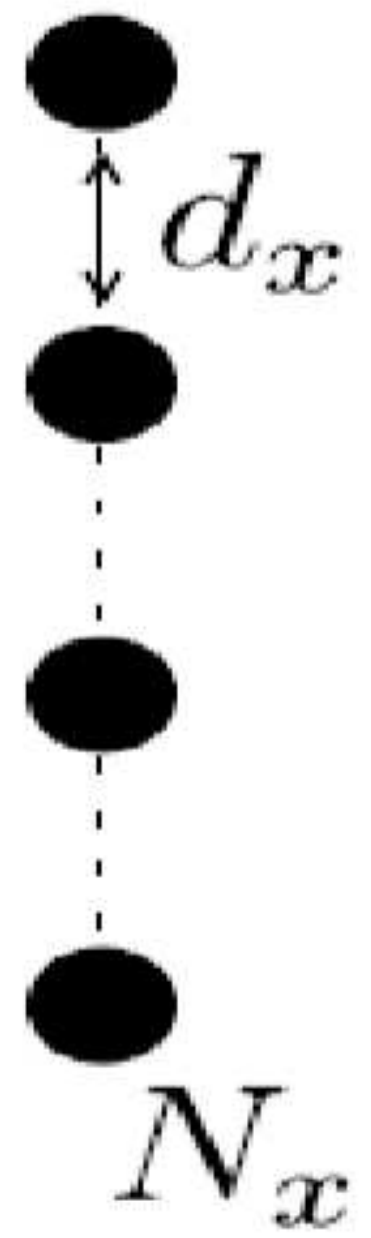

Figure 6

Linear array arrangement 


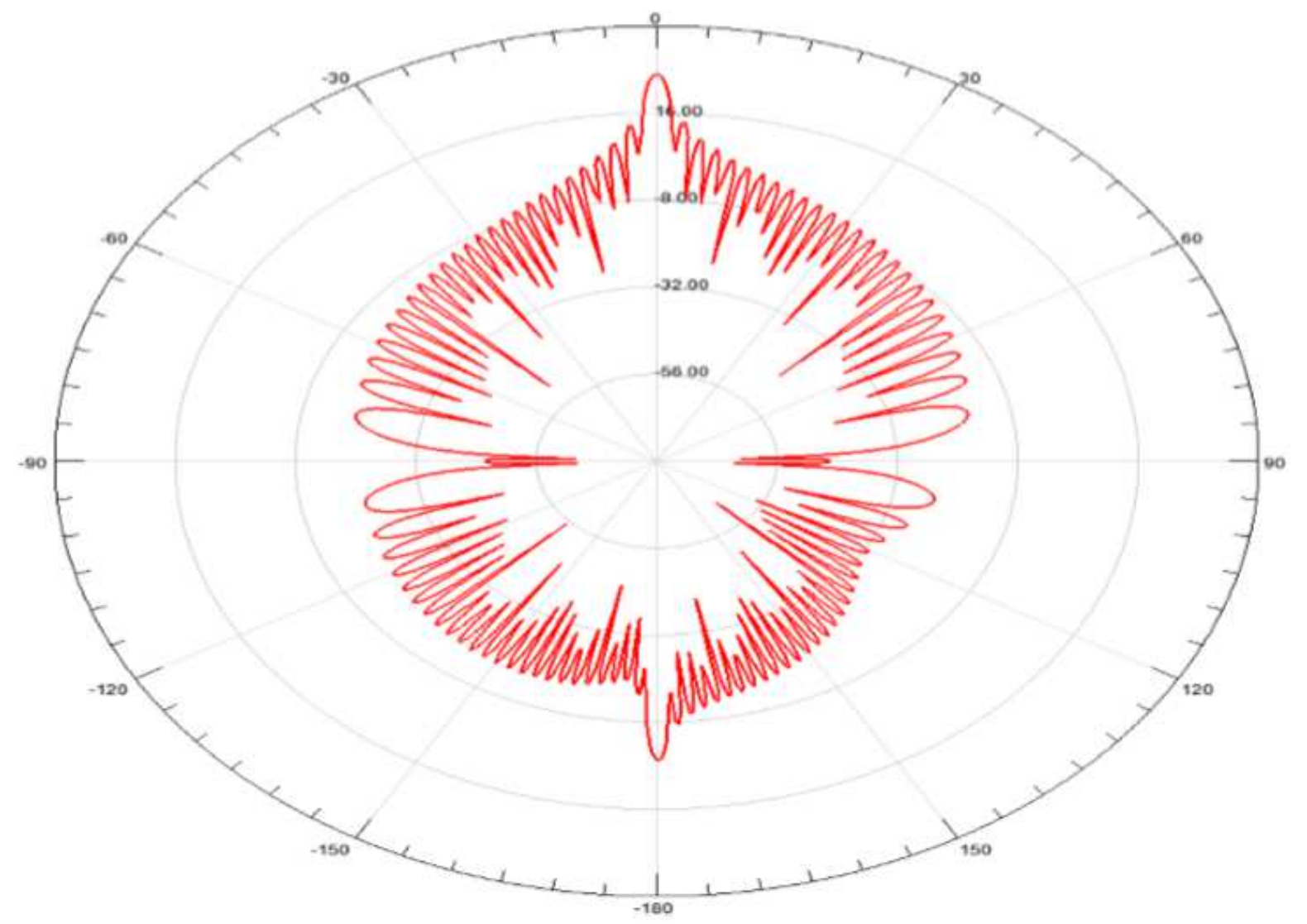

Figure 7

Radiation pattern of the proposed Uniform Linear Array[ $\mathrm{N}=100]$
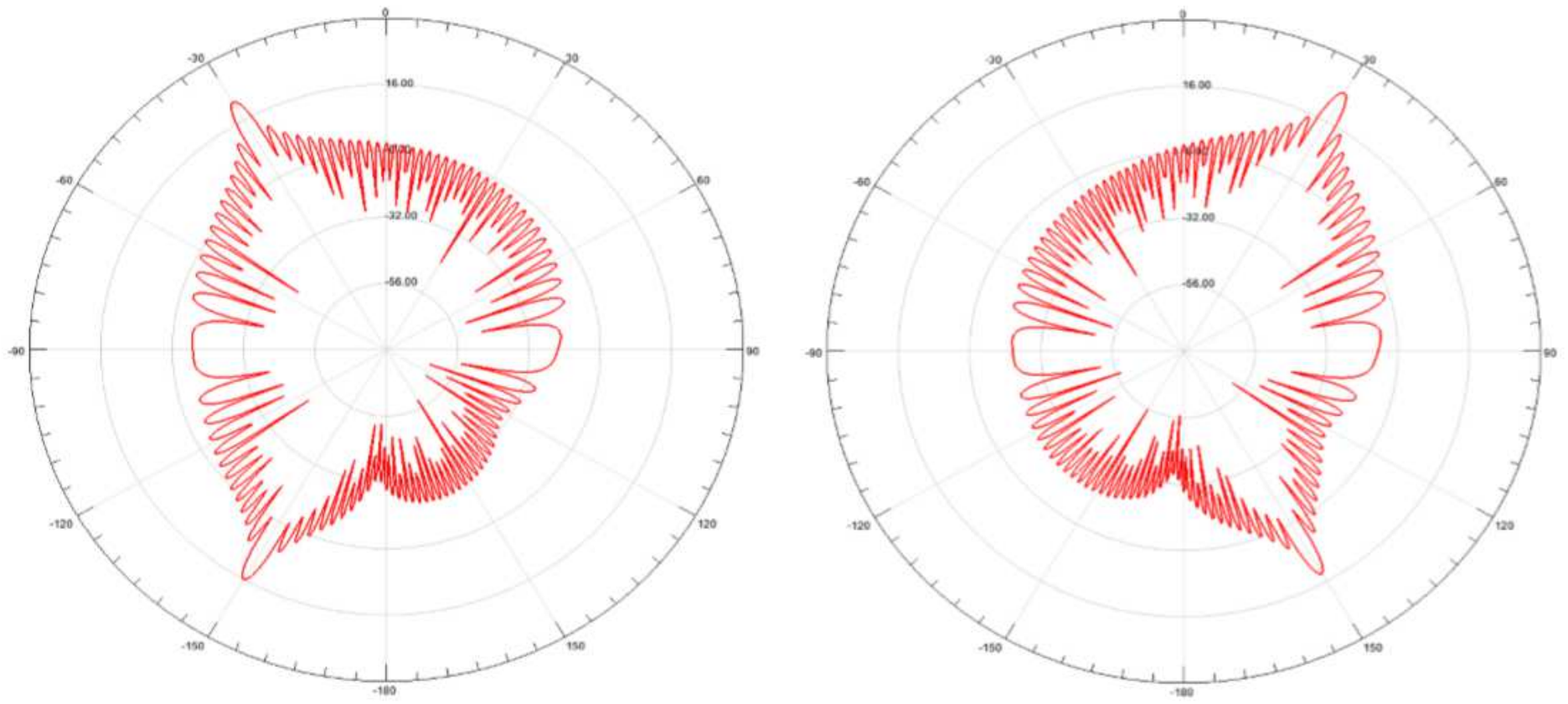

Figure 8 


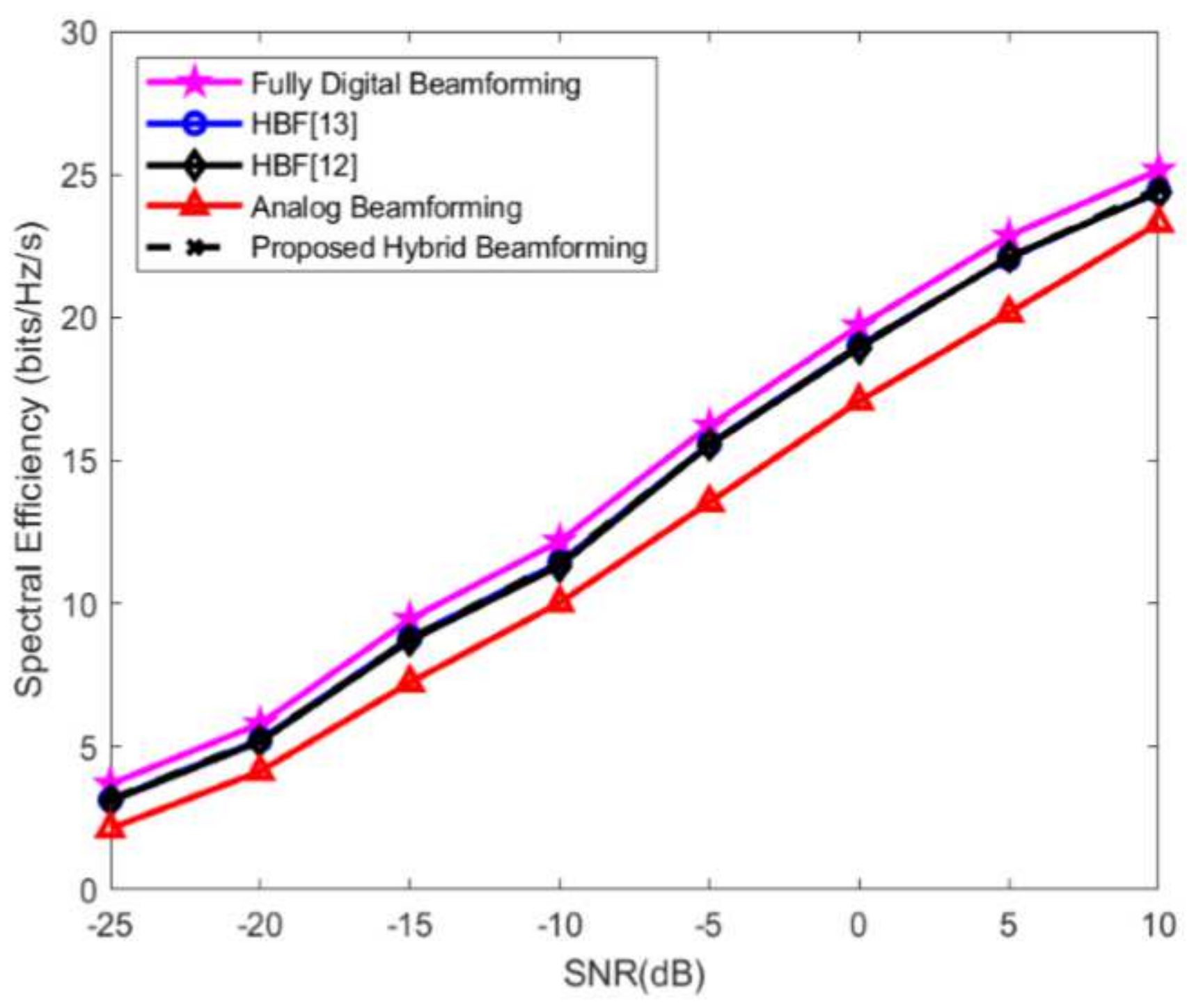

Figure 9

pectral efficiencies achieved by the different beamforming algorithm in a 100×100 mmWave system with $2 \mathrm{RF}$ chains at both the transmitter and receiver. 


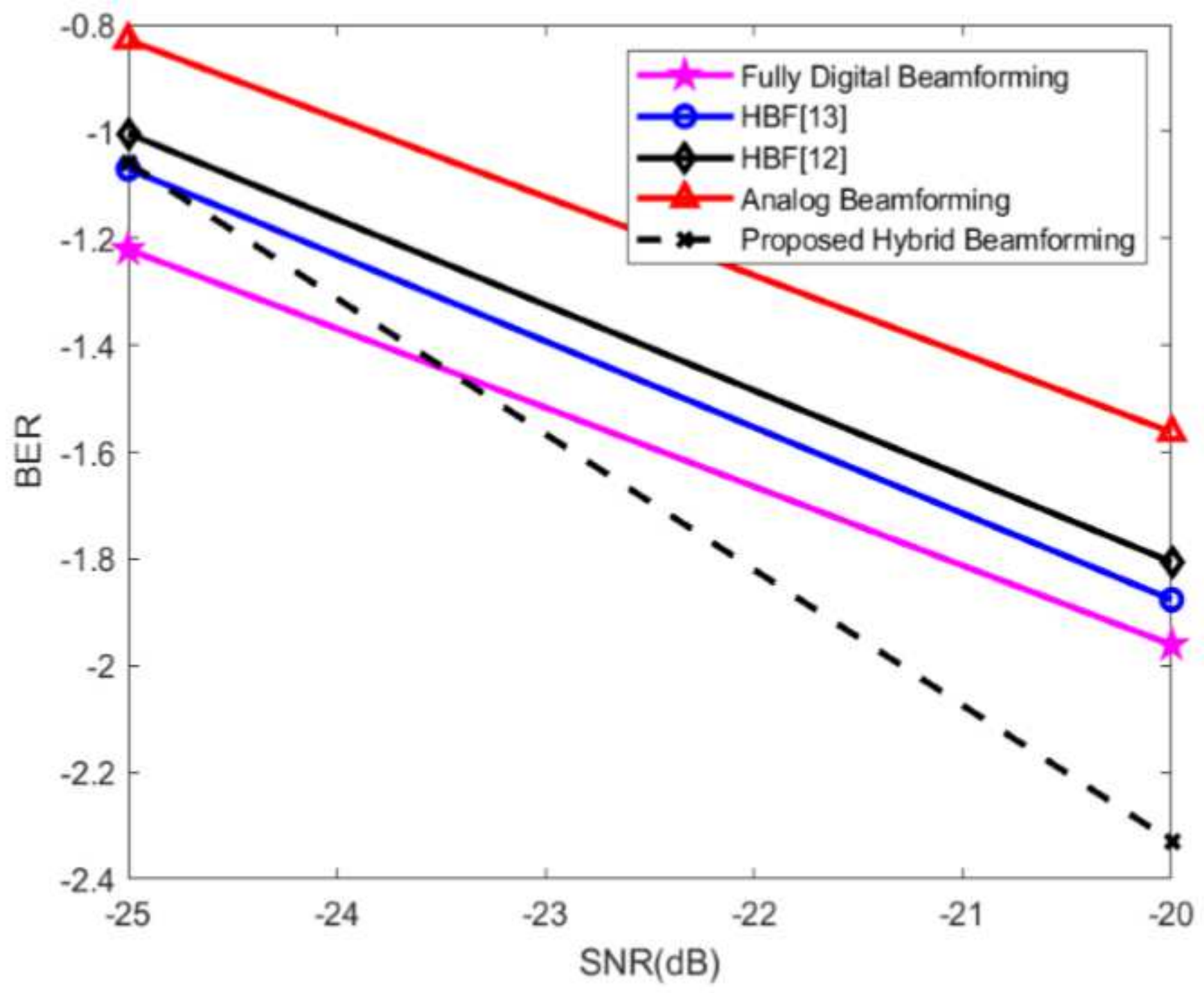

Figure 10

BER achieved by the different beamforming algorithm in a $100 \times 100 \mathrm{mmWave}$ system with 2 RF chains at both the transmitter and receiver. 


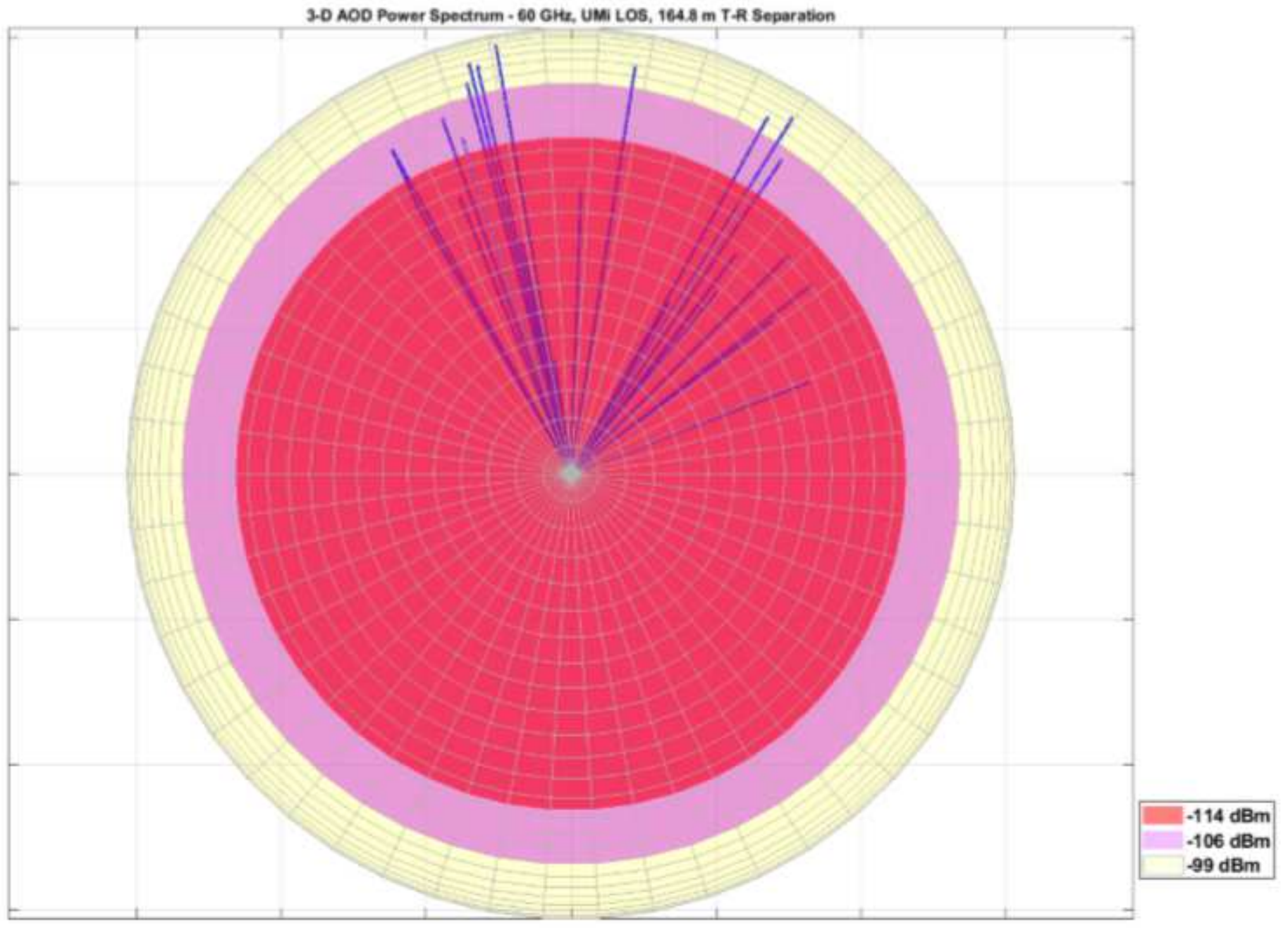

Figure 11

Simulated 3D-AOD power spectrum. 


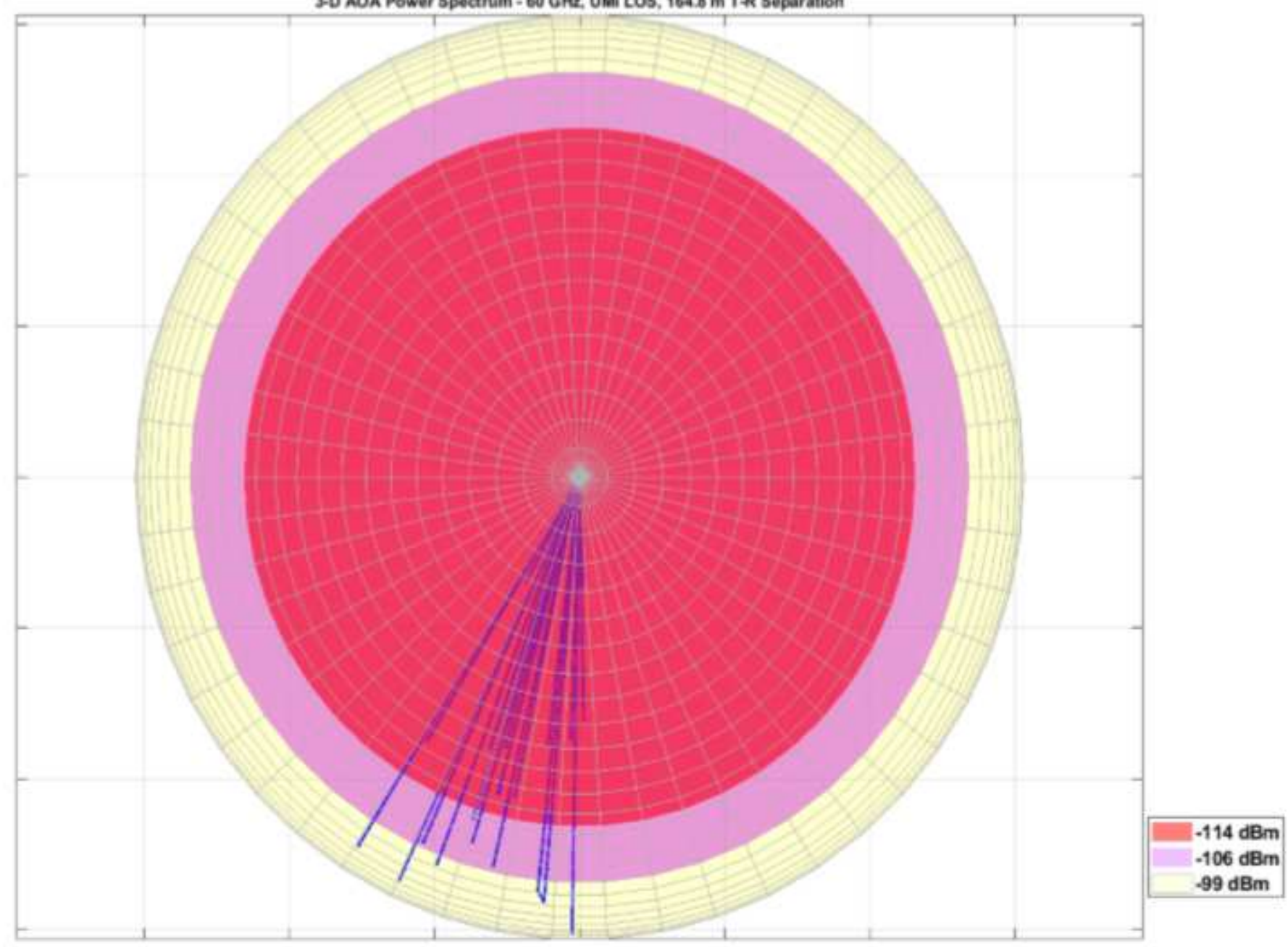

Figure 12

Simulated 3D-AOA power spectrum. 


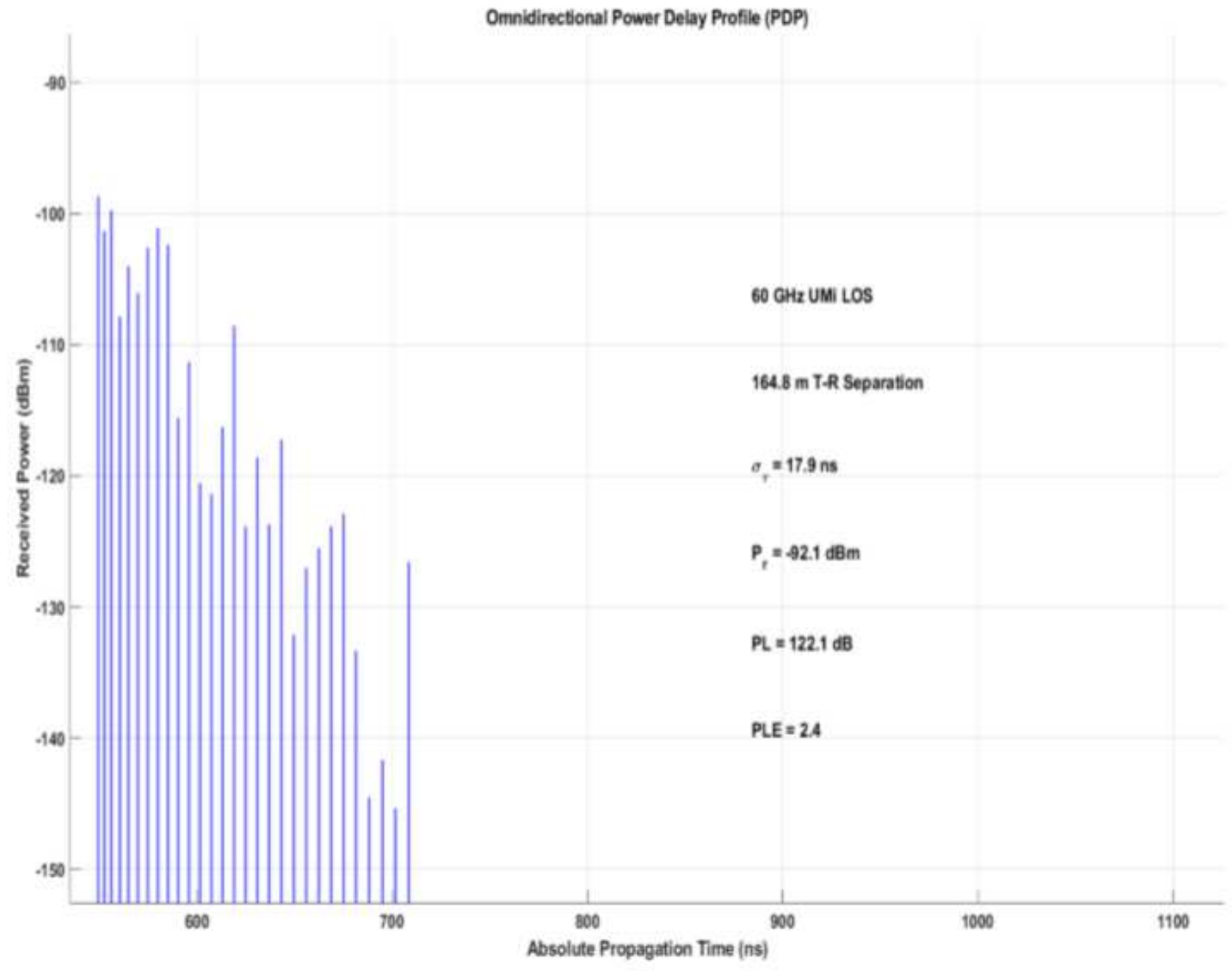

Figure 13

Simulated omnidirectional Power Delay Profile. 


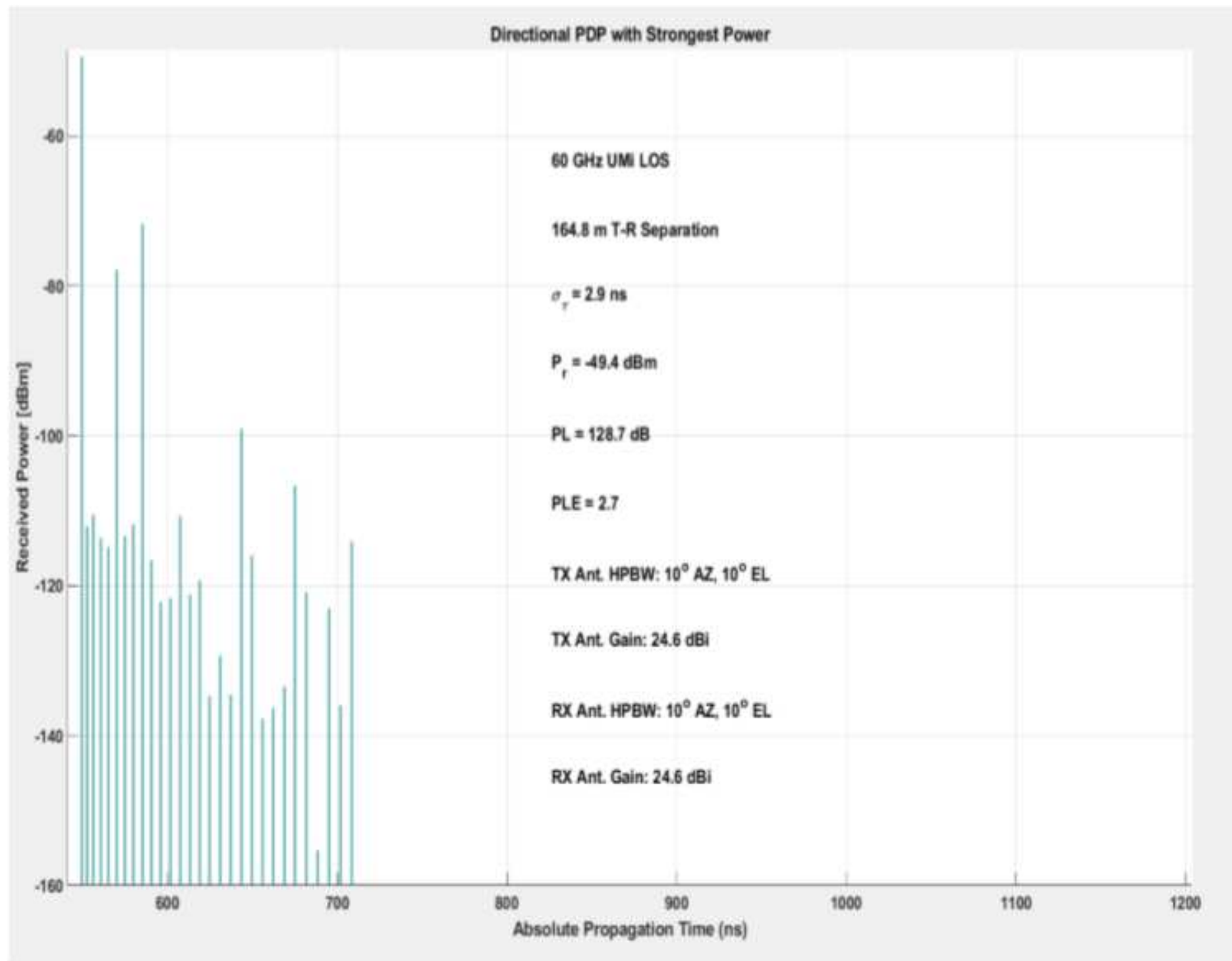

Figure 14

Simulated Directional Power Delay Profile with Strongest Power. 


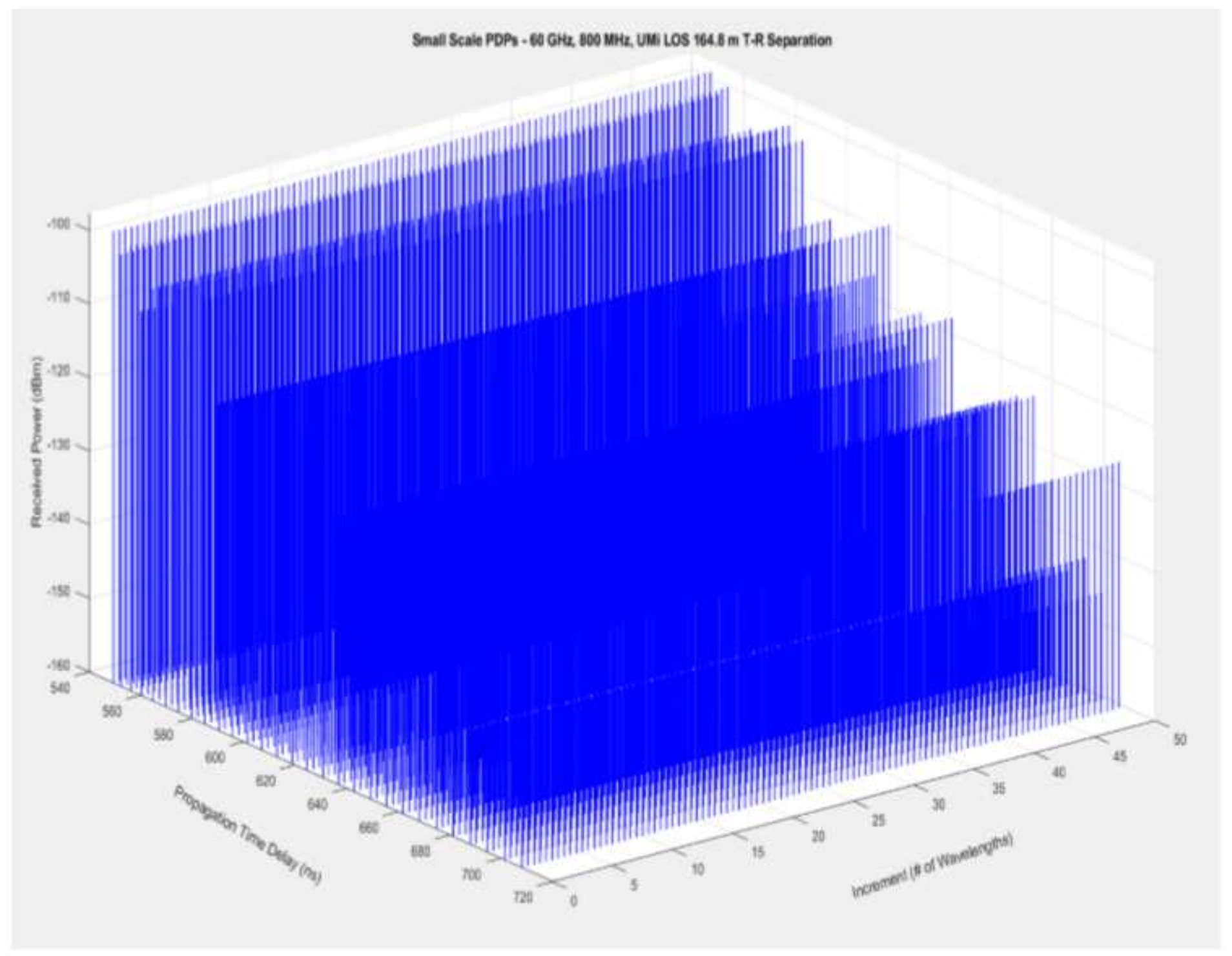

Figure 15

Small Scale power delay profile at $60 \mathrm{GHz}$ RF Bandwidth of $800 \mathrm{MHz}$. 


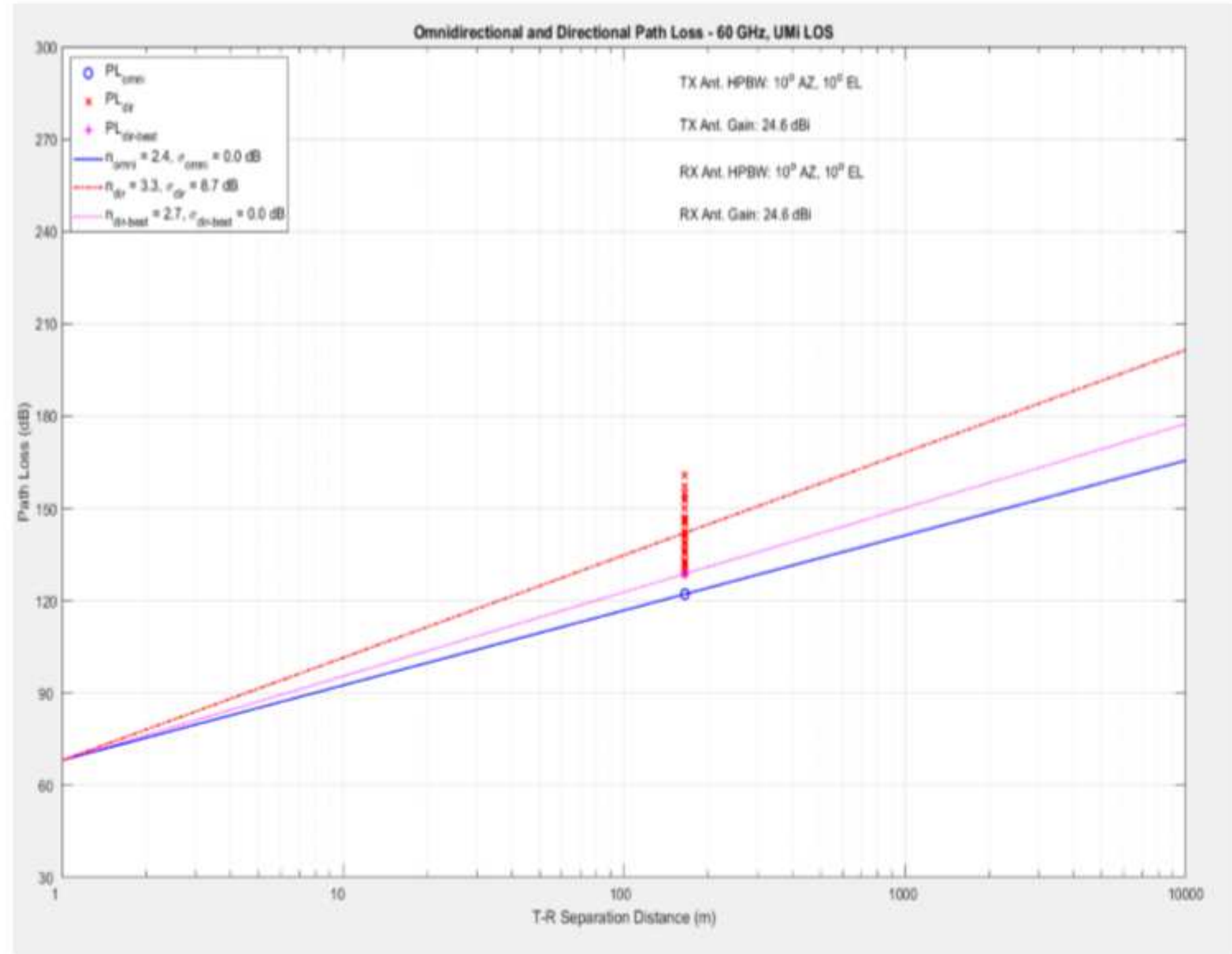

Figure 16

The directional and omni-directional path loss with 100 simulation runs 